\title{
8
}

\section{Globular Cluster X-ray Sources}

\author{
Frank Verbunt \\ Astronomical Institute, Postbox 80.000, 3508 TA Utrecht, The Netherlands \\ Walter H.G. Lewin \\ Massachusetts Institute of Technology, Physics Department, Center for Space Research \\ Cambridge, MA 02139, USA
}

\subsection{Introduction: Some historical remarks}

The earliest detections of luminous X-ray sources $\left(L_{\mathrm{X}} \gtrsim 10^{36} \mathrm{erg} \mathrm{s}^{-1}\right)$ in globular clusters were made with the Uhuru and OSO-7 Observatories (Giacconi et al. 1972 \& 1974; Clark, Markert \& Li, 1975; Canizares \& Neighbours, 1975). About $10 \%$ of the luminous X-ray sources in our Galaxy are found in globular clusters. This implies that the probability (per unit mass) of finding a luminous X-ray source in a globular cluster is about two to three orders of magnitude higher than of finding one in the rest of our galaxy (Gursky 1973, Katz 1975). Clearly, the conditions in globular clusters are very special in that they must be very efficient breeding grounds for X-ray binaries. For reviews which reflect the ideas in the late seventies and early eighties, see Lewin (1980), Lewin \& Joss (1983), Van den Heuvel (1983) and Verbunt \& Hut (1987). At that time there was no evidence for a substantial population of binaries in globular clusters; e.g. Gunn \& Griffin (1979) did not find a single binary in a spectroscopic search for radial velocity variations of 111 bright stars in M 3 .

Clark (1975) suggested that the luminous cluster sources are binaries formed by capture from the remnants of massive stars. Fabian, Pringle \& Rees (1975) specified that they are formed via tidal capture of neutron stars in close encounters with main-sequence stars. Sutantyo (1975) suggested direct collisions between giants and neutron stars as a formation mechanism. Hills (1976) examined the formation of binary systems through star-exchange interactions between neutron stars and primordial binaries of low-mass stars. Hut \& Verbunt (1983) compared the relative efficiencies of tidal capture and exchange encounters for neutron stars and for white dwarfs; and showed that the distribution of X-ray sources among globular clusters with different central densities and core sizes is compatible with the formation by close encounters (Verbunt \& Hut 1987). The importance of mass segregation, which drives the neutron stars to the core, thereby enhancing the capture rate, was demonstrated by Verbunt \& Meylan (1988).

As can be seen from the discovery references in Table 8.1, five luminous globular cluster X-ray sources were known by 1975 , eight by 1980 , ten by 1982 , and thirteen to date. Twelve of these have shown type I X-ray bursts. Measurements of the blackbody radii of the burst sources indicated that they are neutron stars (Swank et al. 1977; Hoffman, Lewin \& Doty 1977a \& 1977b; Van Paradijs 1978). For a review, see Lewin, Van Paradijs \& Taam (1995); see also $\S 3$. The absence of luminous accreting black holes in clusters of our galaxy is presumably a consequence of the small total 

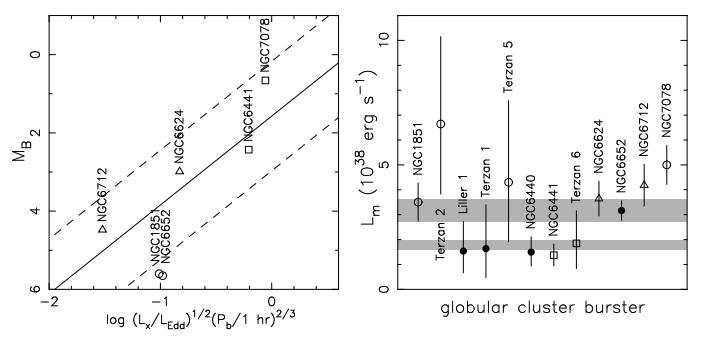

Fig. 8.1. Various types of X-ray sources in globular clusters; sg, $m s$, wd, and ns stand for subgiant, main-sequence star, white dwarf, and neutron star, respectively. From top to bottom: luminous low-mass X-ray binary, lowluminosity low-mass X-ray binary, recycled radio pulsar (here with a white dwarf companion), cataclysmic variable, and magnetically active binary. $L_{s d}$ stands for spin-down luminosity. Approximate maximum luminosities (in the 0.5-4.5 keV range) are indicated on the right. The low-mass X-ray binaries harboring a neutron star are referred to as $L M X B^{\mathrm{NS}}$; when they harbor a back hole, we refer to them as $L M X B^{\mathrm{B} H}$, and we refer to both groups together as $L M X B$.

number of sources, as discussed in 8.5 .1 .2 There is growing evidence that black-hole binaries may exist in globular clusters in several elliptical galaxies (see $§ 8.3$ ).

Because of the observed correlation between the occurrence of a luminous X-ray source in a globular cluster and a high central density, it was expected already early on that these luminous sources would be located close to the cluster centers. These expectations were confirmed by measurements, carried out with the SAS-3 X-ray Observatory, which showed that the positional error circles with radii of 20$30 \operatorname{arcsec}(90 \%$ confidence) included the optical centers of the clusters (Jernigan \& Clark 1979). Later work with the Einstein observatory greatly refined the positional measurements (Grindlay et al. 1984). Bahcall \& Wolf (1976) have shown that under certain assumptions, the average mass of the X-ray sources can be derived from their positions with respect to the cluster center. Even if one accepts the assumptions made, the average mass derived this way for the luminous X-ray sources in globular clusters was not sufficiently accurate to classify these sources, but the result was consistent with the earlier conclusions (see e.g., Lewin 1980; Lewin \& Joss 1983) that these are accreting neutron stars (Grindlay et al. 1984).

Sources with $L_{x} \lesssim 10^{35} \mathrm{erg} \mathrm{s}^{-1}$ were first found in globular clusters with Einstein (Hertz \& Grindlay 1983). More were found with ROSAT, by a variety of authors (Table8.2); a final, homogeneous analysis of the complete ROSAT data was made by Verbunt (2001). On the basis of these Einstein and ROSAT results, it has gradually become clear that these sources are a mix of various types (see Figure8.1). Hertz \& Grindlay (1983) suggested that they were mainly cataclysmic variables, and noted that the low-luminosity source in NGC 6440 could be the quiescent counterpart of the luminous transient source in that cluster. Verbunt et al. (1984) argued that the more luminous of the low-luminosity sources are all quiescent low-mass X-ray binaries. The first radio pulsar detected as a low-luminosity source in a globular 


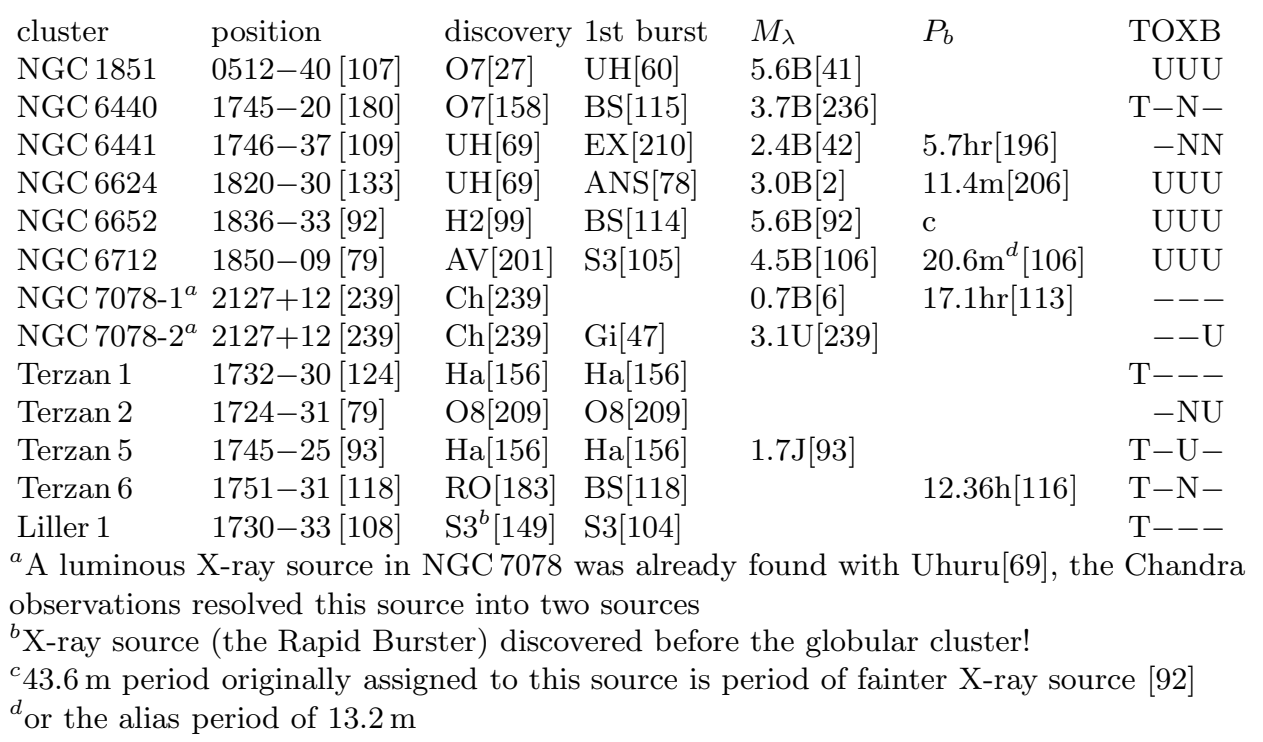

Table 8.1. Some information on the luminous $X$-ray sources in globular clusters of our galaxy. Columns from left to right (1) cluster, (2) rough position (B1950, often used as source name) with reference to the currently most accurate position, (3) the satellite with which the source was discovered as a cluster source, (4) the satellite which detected the first type I X-ray burst from the source, (5) absolute magnitude with filter of optical counterpart, (6) orbital period, (7) indication (with a "T") whether the source is a transient. The last three columns indicate whether a normal $(N)$ or ultrashort $(U)$ orbital period is suggested by the comparison of optical with $X$-ray luminosity (column 8, under "O"), the X-ray spectrum (column 9, under " $X ")$ and the maximum flux reached during bursts (column 10, under " $B$ "). A "-" in columns 8-10 indicates that no information is available. Satellite names are abbreviated as $\mathrm{O}(\mathrm{SO}-)^{2}$ 7, O(SO-)8, UH(URU), H(EAO-)2, A(riel-) V, Ch(andra), Ha(kucho), RO(SAT), S(AS-)3, B(eppo)S(ax), EX(OSAT), Gi(nga). Note that the absolute magnitudes are subject to uncertainties in distance and reddening; also most sources are variable (see Deutsch et al. 2000).

cluster is the pulsar in NGC 6626 (M28, Saito et al. 1997). Finally, Bailyn et al. (1990) pointed out that magnetically active binaries also reach X-ray luminosities in the range of the less luminous sources detected with ROSAT.

It was also realized that some of the sources could be unresolved multiple sources; and unresolved emission was found e.g. by Fox et al. (1996) in NGC 6341 and NGC 6205. However, it is fair to say that the actual plethora of sources shown by the Chandra observations in virtually every cluster that it observed (Tables 8.2 . 8.4) was unpredicted. These observations confirmed that quiescent low-mass X-ray binaries, cataclysmic variables, pulsars, and magnetically active binaries are all Xray sources in globular clusters, as is discussed in 8.4 Whereas some of the Einstein 
and ROSAT sources are confirmed with Chandra as single sources, others have been resolved into multiple sources; details are given in Table 8.2.

The positions obtained with ROSAT were sufficiently accurate to find plausible optical counterparts in Hubble Space Telescope (HST) observations in a number of cases. This work was pioneered in NGC 6397 with a search for $H \alpha$ emitting objects by Cool et al. $(1993,1995)$, and spectroscopic follow-up confirming the classification as cataclysmic variables by Grindlay et al. (1995), Cool et al. (1998) and Edmonds et al. (1999). Plausible candidate counterparts were also found for two X-ray sources in the core of $\omega$ Cen (Carson et al. 2000). All of these suggested counterparts were confirmed with the more accurate positions obtained with Chandra. In 47 Tuc, of the candidate counterparts suggested by a variety of authors, Verbunt \& Hasinger (1998) only retain three, on the basis of more accurate positions of the X-ray sources; these were also confirmed with Chandra. Ferraro et al. (1997) suggested ultraviolet stars as counterparts for two sources found by Fox et al. (1996) in NGC 6205 (see also Verbunt 2001). An ultraviolet counterpart suggested by Ferraro et al. (2000) for a source in NGC 6341 is incompatible with the position of that source (Geffert 1998, Verbunt 2001). Another approach is to look for X-rays from an already known special object. A dwarf nova known since 1941 well outside the central region of NGC 5904 (Oosterhoff 1941) was detected with ROSAT (Hakala et al. 1997), and a pulsar in M28 (Lyne et al. 1987) was detected with ASCA (Saito et al. 1997). Before Chandra, no magnetically active binary was suggested as an optical counterpart for a specific X-ray source.

The luminous X-ray sources in globular clusters are binary systems, and most (if not all) of the low-luminosity X-ray sources are also binary systems or have evolved from them. The presence of binaries is a very important factor in the evolution of a globular cluster (Hut et al. 1992). Theoretical considerations and numerical calculations show that a cluster of single stars is unstable against collapse of its core (Hénon 1961). If binaries are present, however, close binary-single star encounters can increase the velocity of the single stars by shrinking the binary orbits. Binaries can therefore become a substantial source of energy for the cluster, sufficient even to reverse the core collapse. Even a handful of very close binaries can significantly modify the evolution of a globular cluster (Goodman \& Hut, 1989). With a million stars in the cluster as a whole, the number of stars in the core of a collapsed cluster may be only a few thousand. A close binary system, such as an X-ray binary, will have a binding energy that can easily be a few hundred times larger than the kinetic energy of a single star. A dozen such systems, as they were formed, released an amount of energy that is comparable to the kinetic energy of the core as a whole. Encounters between such binaries and other single stars or binaries have the potential to change the state of the core dramatically by increasing or decreasing the core size, and by kicking stars and binaries into the cluster halo or even out of the cluster altogether. The study of the binaries, and X-ray binaries in particular, is therefore of great importance as they play a key role in the cluster's dynamical evolution.

It has been suggested that globular clusters are responsible for the formation of all or some of the low-mass X-ray binaries in our Galaxy, also those outside clusters now (e.g. Grindlay \& Hertz 1985). Specifically, such an origin was suggested by Mirabel et al. (2001) for the black-hole X-ray binary XTEJ1118+480, and by Mirabel \& 


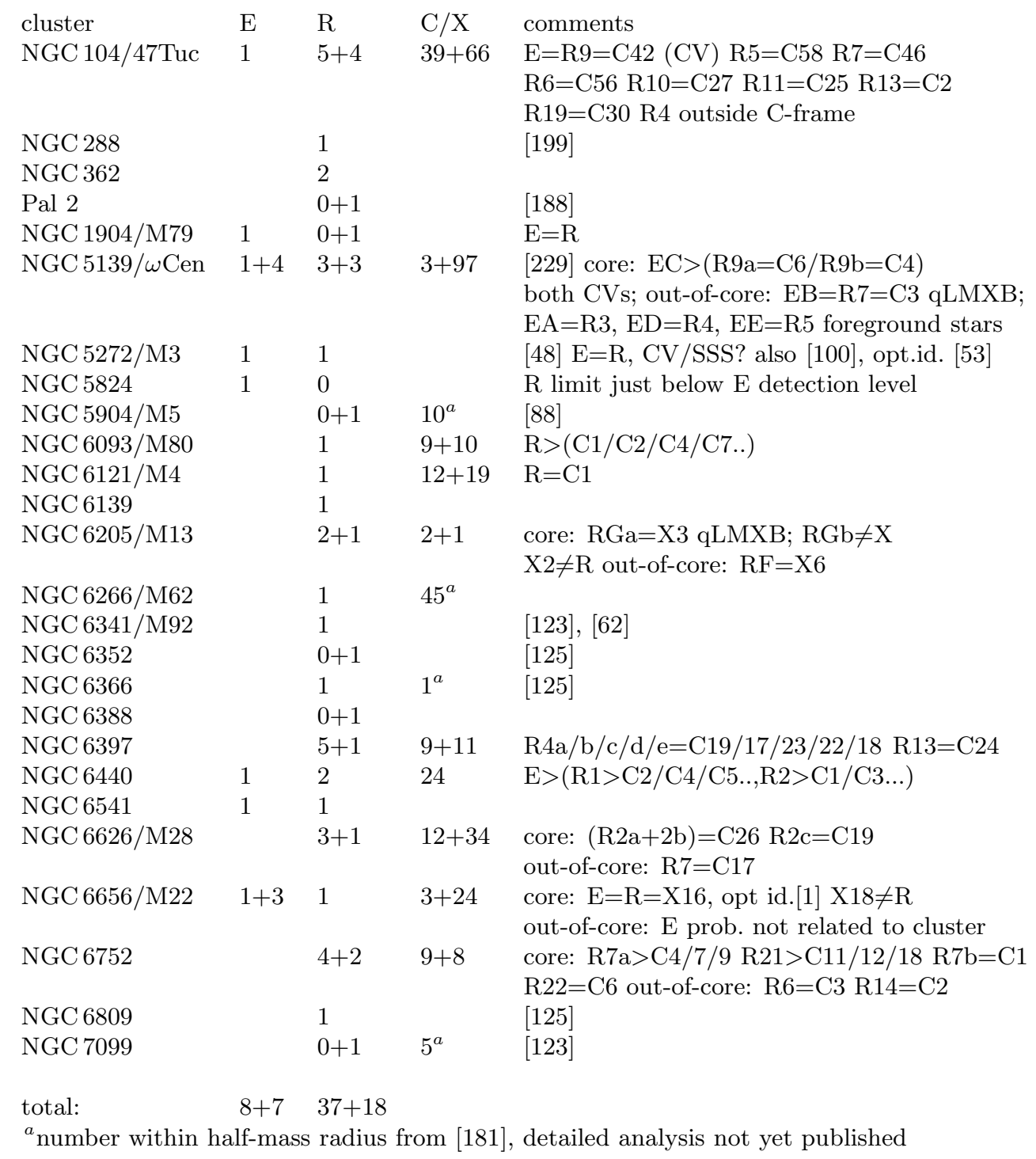

Table 8.2. Observations of low-luminosity sources in globular clusters. We list the number of sources found with Einstein (under E), ROSAT ( $R$ ) and Chandra or $X M M(C / X)$. Numbers following the + sign indicate sources outside the cluster core. Note that the detection limits are very different between clusters. References are Hertz $\&$ Grindlay (1983) for Einstein sources, Verbunt (2001) and references therein for ROSAT sources. References for Chandra and XMM-Newton are listed in Table8.4. Under comments we provide additional references for ROSAT, give occasional source types quiescent (i.e. low-luminosity) low-mass $X$-ray binary $(q L M X B)$ and cataclysmic variable $(C V)$, and indicate the relation between sources observed by subsequent satellites. $=$ identical; $>$ resolved into multiple sources; $A \neq B$ source $A$ not detected by satellite $B$, due to significant variability. Source numbers under comments are those in the references given. 
Rodrigues (2003) for Sco X-1, on the basis of their orbits in the Galaxy. The discovery of very large populations of cluster X-ray sources in other galaxies has rekindled the question of cluster origin for non-cluster sources. In 8.3 .5 we will give the reasons why we believe that most X-ray binaries in the disk of our Galaxy were formed there, and not in globular clusters.

\subsection{The luminous globular cluster X-ray sources in the Galaxy}

In Table 8.1, we list some information on the 13 luminous globular cluster

X-ray binaries in the Galaxy. A comprehensive study of the X-ray spectra of these luminous sources is made by Sidoli et al. (2001), who used BeppoSAX observations in the spectral range between 0.1 and $100 \mathrm{keV}$. They find that the luminous sources in NGC 1851, NGC 6712 and NGC 6624 have similar spectra. When a two-component model (the sum of a disk-blackbody and a Comptonized spectrum) is used to describe the spectrum, the fitted radii and temperatures are compatible with values expected for radii and temperatures of the inner disk. The spectrum of the luminous source in NGC 6652 is similar, except that some radiation is blocked, possibly by the outer disk (Parmar et al. 2001). The spectra of the luminous sources in NGC 6440, NGC 6441, Terzan 2 and Terzan 6 are very different. In the two-component model the inner disk temperature was higher than that of the seed spectrum injected into the Comptonizing plasma, and the inner radius was smaller than those of realistic neutron-star radii. BeppoSAX observed the Rapid Burster in Liller 1 and the luminous source in Terzan 1 when these sources were in a low state; the two luminous sources in NGC 7078 could not be resolved.

Sidoli et al. (2001) suggest, on the basis of binary systems whose orbital periods are known (see Table 8.1), that the two types of spectra correspond to two types of orbital periods: the ultrashort-period systems (observed in NGC 6712 and NGC 6624) and the longer/normal period systems (observed in NGC 6441 and NGC 7078-1). We classify the sources as ultrashort (orbital period less than $60 \mathrm{~m}$, say) or normal based on this correspondence, in Table 8.1 column (9). It may be noted that this classification does not depend on the physical interpretation of the spectra. The luminous source in Terzan 5 has been added to the suggested ultrashort-period systems, on the basis of its X-ray spectrum as observed with Chandra (Heinke et al. 2003a).

It is interesting to compare this tentative classification with two others. The first of these is based on the finding that ultrashort-period systems have a much lower ratio of optical to X-ray flux than systems with longer periods: the optical flux is due to reprocessing of X-rays in the accretion disk; a small accretion disk has therefore a small optical flux (Van Paradijs \& McClintock 1994). Thus the absolute visual magnitude, in conjunction with the X-ray luminosity, may be used to estimate whether the orbital period is ultrashort or not. This is done in column (8) of Table 8.1] The other tentative classification scheme is based on the notion that the white dwarf donor stars in ultrashort-period systems do not contain hydrogen. The X-ray bursts of hydrogen-free matter can reach higher luminosities because the Eddington limit is higher in the absence of hydrogen. Kuulkers et al. (2003) have carefully investigated the maximum observed luminosities of bursters in globular clusters. On this basis we can also tentatively classify ultrashort-period systems, as we have done in column(10) of Table 8.1 


\subsection{The luminous globular cluster $X$-ray sources in the Galaxy 7}

It is seen that the different classifications are consistent for known ultrashort-period systems in NGC 6624 and NGC 6712 and tentatively classified ultrashort systems in NGC 1851 and NGC 6652 and for the systems with known longer period in NGC 6441 and Terzan 6 . Two tentative indicators for the source in Terzan 2 are contradictory.

Five of the thirteen luminous X-ray sources are transients. The source in Terzan 1 has been consistently luminous until about 1999, when it switched off (Guainazzi et al. 1999). The Rapid Burster in Liller 1 and the luminous source in Terzan 6 are recurrent transients, showing outbursts quite frequently. Intervals of $\sim 6-8$ months (Lewin et al. 1995) and $\sim 100$ days (Masetti 2002) were observed for the Rapid Burster, and $\sim 4.5$ months for the luminous source in Terzan 6 (in 't Zand et al. 2003). The luminous source in NGC 6440 is a transient whose outbursts have been detected in 1971, 1998 and 2001 (see $\S 8.2 .1$ ). The transient source in Terzan 5 entered a rare high state in August 2000 (Heinke et al. 2003a, and references therein). Interestingly, most (known and suggested) ultrashort-period systems are persistent sources. Note, however, that one of the two periods known for low-luminosity lowmass X-ray binaries is also ultrashort (see Fig. 8.14). (The source in NGC 6652 does occasionally drop below $\sim 10^{36} \mathrm{erg} \mathrm{s}^{-1}$, but it is not known by how much.) Whether the above correlations are significant remains to be seen, and will only become evident once more secure orbital periods have been determined.

With Chandra, the positions of the luminous sources have become more accurate. In Figure 8.2 we show these positions, together with those of the low-luminosity sources that also contain a neutron star. It is seen that some sources, e.g. the luminous source in NGC 6652, are at a large distance from the cluster core.

\subsubsection{Notes on individual sources}

$N G C 1851$. The accurate Chandra position for the luminous source in NGC 1851 confirms the previously suggested optical counterpart; this star is very faint, considering the brightness of the X-ray source, which suggests that the binary is an ultra-short period binary (see $\S 8.2$ and Table 8.1 ), i.e. $P_{b}<1 \mathrm{~h}$ (Homer et al. 2001a).

NGC 6440. The luminous source in NGC 6440 is a transient; outbursts were detected in 1971 with OSO-7 and Uhuru (Markert et al. 1975, Forman et al. 1976), and again in 1998 and 2001 with BeppoSAX (in 't Zand et al. 1999, 2001). The 1998 outburst was followed up with NTT and VLT observations. An optical transient was found at the approximate location of the X-ray transient (Verbunt et al. 2000). The 2001 outburst was observed with Chandra (in 't Zand et al. 2001), and the source was identified with one of four low-luminosity sources found earlier by Pooley et al. (2002b). The 1998 optical and the $2001 \mathrm{X}$-ray transient are the same source.

NGC 6624. The luminous source in NGC 6624 has an orbital period of $865 \mathrm{~s}$, indicating that the donor is a white dwarf (Verbunt 1987). For such a donor, theory predicts that the orbital period increases with time: $\dot{P}_{b} / P_{b}>8.8 \times 10^{-8} \mathrm{yr}^{-1}$. However, observations made in the period 1967 to 1997 show a decrease in the period, of order $\dot{P}_{b} / P_{b}=-5.3 \times 10^{-8} \mathrm{yr}^{-1}$ (Van der Klis et al. 1993, Chou \& Grindlay 2001). This continued decrease cannot be explained by changes in the disk size. However, the X-ray source is located close to the center of the cluster (King et al. 1993), and if the central density is high enough, acceleration of the binary in the cluster potential may explain the difference (Chou \& Grindlay 2001). Further study is required as 


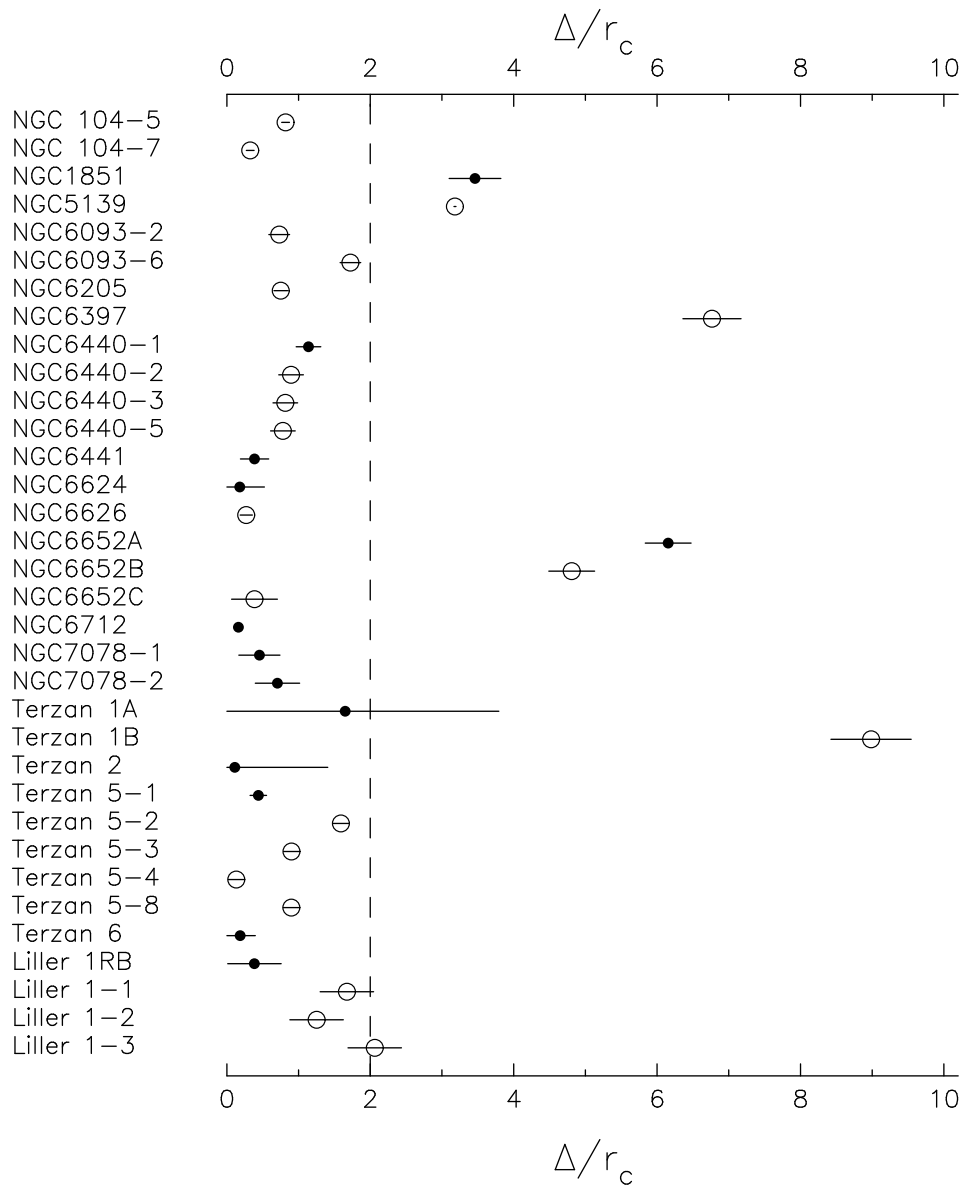

Fig. 8.2. Distance $\Delta$ of Low-Mass X-ray Binaries to the center of the globular cluster in which they are located, in units of the core radius $r_{c}$. Luminous and lowluminosity sources are indicated with $\bullet$ and $\circ$, respectively. Errors are computed from the uncertainty in the X-ray position and from the uncertainty in the position of the cluster center (assumed to be $1.2^{\prime \prime}$ ). Core radii and centers are taken from Harris (1996, February 2003 version), except for Terzan 6 (in 't Zand et al. 2003). References for the X-ray positions are in Tables8.1 and 8.2 It is seen that most, but not all, X-ray binaries are within $2 r_{c}$.

discrepancies exist between reported positions for the cluster's center. It is important that the central density of the cluster be determined more accurately. A viable alternative may be that the donor is not a white dwarf, but a stripped core of a slightly evolved main-sequence star (Podsiadlowski, Rappaport \& Pfahl 2002).

NGC 6652. Chandra observations of NGC 6652 show three low-luminosity sources in addition to the luminous source. The optical counterpart with a $43.6 \mathrm{~m}$ orbital period previously suggested (Deutsch et al. 2000) for the luminous source turns out to be one of the low-luminosity sources instead (Heinke et al. 2001). The Chandra data were obtained with the High Resolution Camera and thus do not contain much 


\subsection{The luminous globular cluster $X$-ray sources in the Galaxy 9}

spectral information. The visual brightness of the new optical counterpart of the luminous X-ray source is still very low; and the suggestion (Deutsch et al. 2000) that this source is an ultra-short period binary stands (see Table 8.1).

$N G C 7078$. A Chandra observation of NGC 7078 (M15) showed that this cluster contains two luminous sources, at a separation of $3^{\prime \prime}$, seen as a single source in earlier observations with instruments that have less spatial resolution (White \& Angelini 2001). The presence of two sources actually had been predicted by Grindlay (1992), as a solution to a puzzle posed by previous observations. The high optical to X-ray flux ratio indicated that the central X-ray source is hidden by the accretion disk, and that only X-rays scattered in our direction by a corona are detected; this implies that the intrinsic X-ray luminosity exceeds the observed luminosity by almost two orders of magnitude (Aurière et al. 1984). However, burst observations indicated that the bursts reached the Eddington limit for the distance to M15; this implied that there was no blockage of radiation, and thus that the observed persistent flux was representative for the full luminosity (Dotani et al. 1990). The brightest of the two (7078-2, see Table 8.1) is the burster; the optical counterpart is probably a blue star with $U=18.6$; its position is determined most accurately from its radio counterpart (Kulkarni et al. 1990). The less luminous source 7078-1 has the disk corona, and is identified optically with a $17.1 \mathrm{hr}$ partially eclipsing binary (Ilovaisky et al. 1993). Its optical brightness and the orbital period - revealed by variable, non-total eclipses

- indicate that the donor in this system is a sub-giant. Ultraviolet lines with strong P Cygni profiles indicate extensive mass loss. An analysis of the eclipse timing puts a rough upper limit on the period change of $0.01 \mathrm{~d}$ in $22 \mathrm{yr}$ (Naylor et al. 1992; Ioannou et al. 2003). An extreme ultraviolet flux has been detected from M15. It was believed to come from the X-ray binary AC211, the optical counterpart of 7078-1 (Callanan et al. 1999). We suggest that some UV may also come from 7078-2 which allows for a direct view to the center of the accretion disk.

Terzan 1. When Terzan 1 was observed with BeppoSAX in April 1999, the luminosity had dropped to about $2 \times 10^{33} \mathrm{erg} \mathrm{s}^{-1}$, indicating that the luminous source in this cluster had gone into quiescence (Guainazzi et al. 1999). Accurate positions for the luminous source had been obtained with EXOSAT ( 8 " accuracy, Parmar et al. 1989) and ROSAT (5" accuracy, Johnston et al. 1995); remarkably, the source detected with Chandra is not compatible with these positions (Wijnands et al. 2002). Probably, all observations of the bright state before 1995 refer to the same source, since the detected luminosities are all similar at, or just below, $10^{36} \mathrm{erg} \mathrm{s}^{-1}$ (Skinner et al. 1987; Parmar et al. 1989, Verbunt et al. 1995, Johnston et al. 1995). This source was discovered in 1980 during observations with Hakucho; only two bursts were observed in one week. The upper limit to the persistent flux was $\sim 10^{36} \mathrm{erg} \mathrm{s}^{-1}$ (Makishima et al. 1981). It is not clear whether BeppoSAX detected the faint state of the luminous source, or the low-luminosity source found with Chandra.

Terzan 5. Observations of Terzan 5 with Chandra show nine sources in addition to the transient; four of these are probably low-luminosity $\mathrm{LMXB}^{\mathrm{NS}}$ (Heinke et al. 2003a). A possible optical counterpart is a faint blue (in infrared colors) star, at $M_{J} \simeq 1.7$ when the X-ray source was faint. Heinke et al. (2003a) note that the $\mathrm{X}$-ray spectrum when the source is luminous is like those of the luminous sources in NGC 6624 and NGC 6712, and suggest that the source is an ultra-compact binary 
(see Table 8.1). If that is the case, its high optical flux is surprising. Wijnands et al. (2005) find that the spectrum in quiescence (near $10^{33} \mathrm{erg} \mathrm{s}^{-1}$ ) is dominated by a hard power-law component.

Terzan 6. Extended studies of Terzan 6 with RXTE show that the transient X-ray source in this cluster has fairly frequent outbursts, on average every 140 days (in 't Zand et al. 2003). An X-ray position, derived from a Chandra observation, and an improved position for the center of the cluster, found with ESO NTT observations, show that the X-ray source is close to the cluster center. The RXTE observations provide an upper limit to the change in the orbital period: $|\dot{P} / P|<3 \times 10^{-8} \mathrm{yr}^{-1}$.

Liller 1. The Rapid Burster in Liller 1 is a recurrent transient. It shows a bewildering variety of X-ray behavior. When discovered in 1976 (Lewin et al. 1976), it emitted X-rays largely in the form of very frequent bursts (which were later called type II bursts). The average burst rate was in excess of $10^{3}$ per day; this gave the source its name. There is an approximate linear relation between the burst fluence and the waiting time to the next burst (i.e. the mechanism is like that of a relaxation oscillator). These rapid bursts are the result of spasmodic accretion. Type II bursts have been observed that lasted up to ten minutes with a corresponding waiting time to the next burst of $\sim 1 \mathrm{~h}$. At times (early in an outburst which typically lasts several weeks), for periods of many days, the Rapid Burster behaved like a normal LMXB (i.e., persistent emission, but no type II bursts). The Rapid Burster also produces the thermonuclear, type I, bursts (Hoffman, Marshall \& Lewin, 1978). A review of this remarkable source is given by Lewin, Van Paradijs \& Taam (1993); see also §2.9.5. An accurate Chandra position of the Rapid Burster (Homer et al. 2001b) coincides with the radio counterpart (Moore et al. 2000). The Einstein position of the Rapid Burster (Hertz \& Grindlay 1983) is not compatible with the radio counterpart and with the Chandra position. However, it does coincide with one of three low-luminosity sources also detected with Chandra. Perhaps the low-luminosity source was more luminous at the time of the Einstein observations. On the basis of their luminosities, the low-luminosity sources are probably low-mass X-ray binaries in quiescence (Homer et al. 2001b).

\subsection{The globular cluster sources outside the Galaxy}

In this section, we discuss the very luminous globular cluster X-ray sources observed in galaxies other than our own. The observations we discuss were all done with Chandra, except for the ROSAT observations of M 31. Some of the sources were already detected with ROSAT, but the positional accurracy of Chandra allows more secure identifications with globular clusters. Table 8.3 gives an overview of the observations reported so far. The lowest detectable luminosities vary strongly between galaxies. With the exception of M31 and NGC 5128, however, we are always talking about very luminous sources (the tip of the iceberg). In addition to the sources discussed in this chapter, sources in many other globular clusters associated with other galaxies have been observed but not (yet) recognized as such, e.g. because the required optical cluster studies are not available (see $\S 12$ and Table 12.1).

The number of globular clusters varies widely between galaxies. Precise numbers are difficult to determine: clusters are difficult to detect against the bright background of the central regions of a galaxy, and the cluster distribution may extend 


\begin{tabular}{|c|c|c|c|c|c|c|c|c|c|c|}
\hline & & & & & $\mathrm{HS}$ & $\mathrm{T}-\mathrm{F}$ & & & & \\
\hline galaxy & $X$ & $X_{g}$ & $\mathrm{~N}$ & $S_{N}$ & $X$ & $X_{g}$ & $\mathrm{~N}$ & $S_{N}$ & $L_{l}$ & $L_{u}$ \\
\hline NGC 720121 & 42 & 12 & & 2.2134 & & & & & 38.6 & 40.0 \\
\hline NGC 1316130 & 81 & 5 & & 1.772 & & & & 0.970 & 37.3 & 39.3 \\
\hline NGC 13993 & 214 & & 6450 & 5.146 & 45 & 32 & 678 & & 37.7 & \\
\hline NGC 1407241 & 160 & 88 & & 4.0173 & & & & & & \\
\hline NGC 155319 & 49 & 2 & & 1.45 & & & 1553 & 0.5141 & 38.3 & 39.3 \\
\hline NGC 3115144 & 90 & & & & 36 & 9 & & & & \\
\hline NGC 4365 144 & 149 & & & 5.0134 & 44 & $18^{a}$ & 660 & 2.1141 & & \\
\hline NGC 4472143 & 135 & & 5900 & 3.6191 & 72 & 29 & 825 & & 37.0 & \\
\hline NGC 4486 126 & 174 & & 13450 & 14160 & 98 & 60 & & & 37.2 & 39.0 \\
\hline NGC 4649 187 & 165 & & & 6.9134 & 40 & 20 & 497 & 1.4141 & & \\
\hline NGC 4697198 & 80 & $>8$ & 1100 & 2.5134 & & & & & 37.7 & 39.4 \\
\hline M 31 207] & 353 & 27 & 500 & 1.211 & & & & & 35.5 & 38.3 \\
\hline M 3144 & & & & & 90 & 28 & & & 35.3 & 38.3 \\
\hline NGC 459445 & 122 & 32 & 1900 & 2.1192 & & & & & & \\
\hline NGC 5128 137 & 111 & 33 & & 2.689 & & 29 & & & 36.2 & \\
\hline
\end{tabular}

Table 8.3. X-ray sources associated with globular clusters in galaxies other than our own. For each galaxy we list the total number of $X$-ray sources detected $X$, the number associated with globular clusters $X_{g}$, and the number of globular clusters $N$ with specific frequency $S_{N}$ (Eq.8.1); and the same numbers again in a limited field-of-view (FOV; Hubble Space Telescope observations, usually with the WFPC-2 but with the ACS for M87) where applicable. We also list the logarithm of the $X$-ray luminosity detection limit, $L_{l}$, and the luminosity, $L_{u}$, of the most luminous cluster source, in $\operatorname{erg~s}^{-1}$. Numbers between [] are references.

beyond the observed area. For example, globular clusters in NGC 4697 have only been identified in an annulus from 1.5 to 2.5 arcmin from the center (Fig. 8.5). And even for nearby M 31 "the size of the globular cluster system is embarrassingly uncertain" (Barmby 2003). Estimates of the total number are often based on an uncertain extrapolation of the measured bright part of the globular cluster luminosity function and depend on the availability of multi-color images that go deep enough to probe a significant portion of the luminosity function (Kundu, private communication). In many galaxies the area in which positions of globular clusters are known with sufficient accuracy for comparison with X-ray positions is limited by the field-of-view of HST-WFPC2 observations: an example is seen in Figure 8.4.

The number $N$ of globular clusters of a galaxy is sometimes scaled to the total luminosity of the galaxy (derived from absolute magnitude $M_{V}$ ), as a specific frequency $S_{N}$, defined as (Harris \& van den Bergh 1981):

$$
\log S_{N}=\log N+0.4\left(M_{V}+15\right)
$$

A 'local' specific frequency is often defined for the field-of-view of the HST-WFPC2. The uncertainties in the total number of globular clusters are reflected in large uncertainties of the specific frequencies, and the uncertainty in the distance adds to 


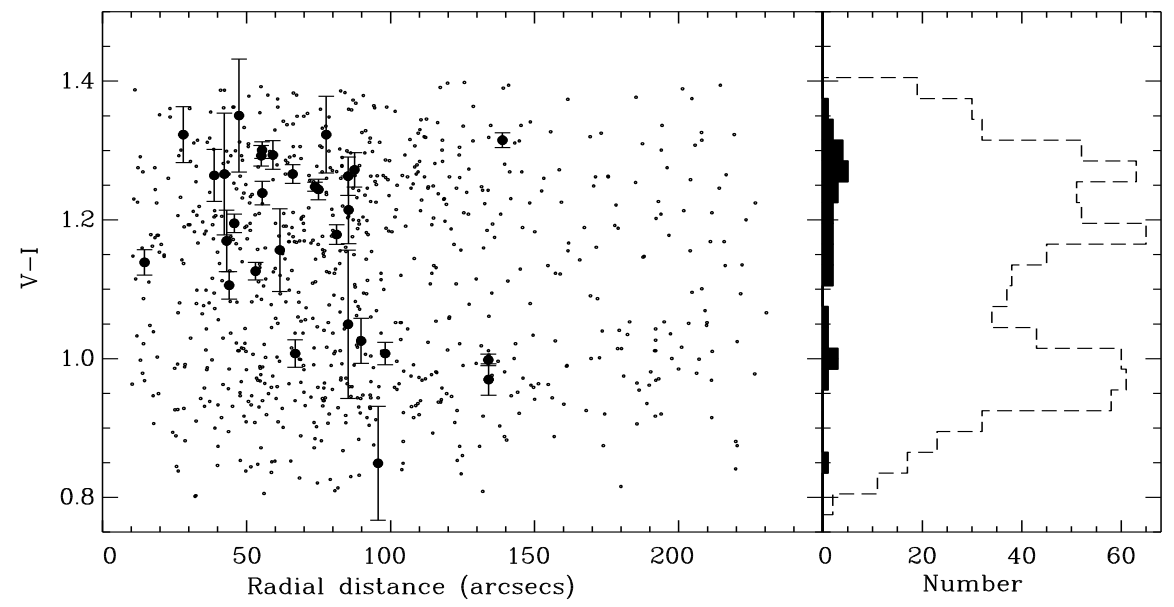

Fig. 8.3. Left: $V-I$ colors of globular clusters vs. distance from the center of the elliptical galaxy NGC 4472. LMXB-globular-cluster matches are indicated by filled circles. Most of the luminous X-ray sources are located in red globular clusters. The optical color distribution is shown on the right with a dashed line; notice the bimodal distribution. The distribution of the globular clusters that house the luminous X-ray sources is also shown. Courtesy of Kundu, Maccarone \& Zepf (2002).

this. For example, values for NGC 1553 range from $1.22 \pm 0.27$ to $2.3 \pm 0.5$ (Bridges \& Hanes 1990, Kissler-Patig 1997). Specific frequencies (most are meant to be global) were compiled by Harris (1991), Kissler-Patig (1997), and Ashman \& Zepf (1998). Local specific frequencies of globular clusters have been measured in the inner region of 60 galaxies (Kundu \& Whitmore, 2001a,b).

Many elliptical galaxies, and especially those in the center of clusters of galaxies, have large numbers of globular clusters (Harris 1991; Ashman \& Zepf 1998). Per unit mass, most ellipticals have about twice as many globular clusters as spirals (Zepf \& Ashman 1993, 1998). The globular cluster populations in most elliptical galaxies show a bimodal distribution in optical colors (Figure 8.3). Most of this is due to differences in metallicity, but differences in age may also play a role. Metal-poor clusters are bluer than metal-rich clusters of the same age; at the same metallicity, old clusters are redder than young ones. It has been suggested that the blue metalpoor globular clusters were formed at the proto-galactic epoch, and that the red metal-rich globular clusters resulted from later starbursts, e.g. as a consequence of the mergers that produce the galaxies that we observe today (Ashman \& Zepf, 1992; Zepf \& Ashman, 1993; for other possibilities see the review by West et al. 2004). However, to date there is no convincing evidence for difference in ages of red and blue subsystems (e.g., Puzia et al. 2002, Cohen et al. 2003, Côté 1999, Côté et al. 2002).

\subsubsection{Elliptical galaxies}

The most luminous X-ray sources in a galaxy are high-mass X-ray binaries, supernova-remnants, and low-mass X-ray binaries. Since elliptical galaxies do not 
house young stellar populations, virtually all luminous X-ray sources in them will be low-mass X-ray binaries. Table 8.3 provides an overview of the references and results; some additional remarks for individual galaxies follow. We will discuss the $\mathrm{X}$-ray luminosity functions of the globular cluster systems and the reported breaks in some of them in $\S 8.3 .4$.

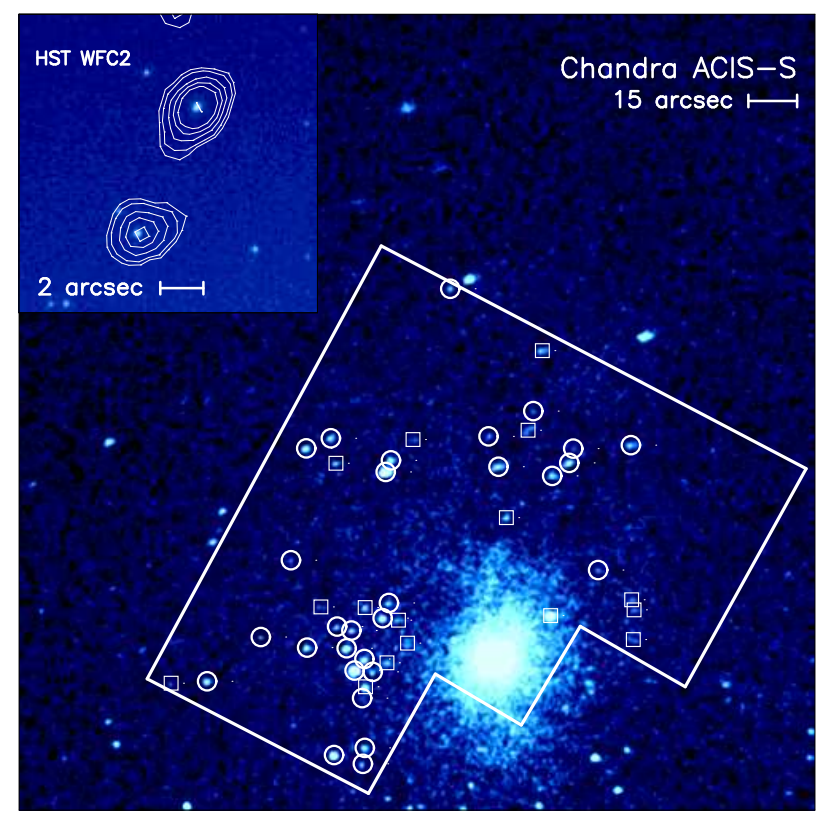

Fig. 8.4. The $0.3-10 \mathrm{keV}$ Chandra image of NGC 1399 centered on an HST pointing, smoothed with a Gaussian of about $0.8^{\prime \prime}$. The white line marks the HST/WFPC2 FOV. The circles show the X-ray source positions that are associated with globular clusters. The squares are the remaining sources. All 45 sources are marked; 38 have a significance in excess of $3 \sigma$. The top left image is an example of the Chandra contours overlaid on the HST field. Courtesy Angelini et al. (2001).

NGC 1399 is a giant elliptical galaxy in the center of the Fornax Cluster at $20.5 \mathrm{Mpc}$. A large fraction of the $2-10 \mathrm{keV} \mathrm{X}$-ray emission in an $8^{\prime} \times 8^{\prime}$ region is resolved into 214 discrete sources, including many background sources. 32 are in globular clusters (see Figure 8.4). Many of the globular cluster sources have superEddington luminosities (for an accreting neutron star), and their average luminosity is higher than that of the sources not associated with globular clusters. The most luminous source in a globular cluster has an ultra-soft spectrum such as seen in the high state of black-hole binaries. This may indicate that some of the most luminous sources are binaries with an accreting black-hole, rather than conglomerates of less luminous neutron-star binaries (Angelini et al. 2001).

Dirsch et al. (2003) find that "within $7^{\prime}$ the specific frequency of the blue clusters alone is a factor $\sim 3$ larger than for the red ones. Outside this radius, both populations have the same high local specific frequency", listed in Table 8.3.

$N G C 4697$. In this galaxy, most of the X-ray emission is from point sources. The 
central source, with $L_{X}=8 \times 10^{38} \mathrm{erg} \mathrm{s}^{-1}$, may be an active nucleus and/or multiple LMXBs (Sarazin et al. 2000, 2001).

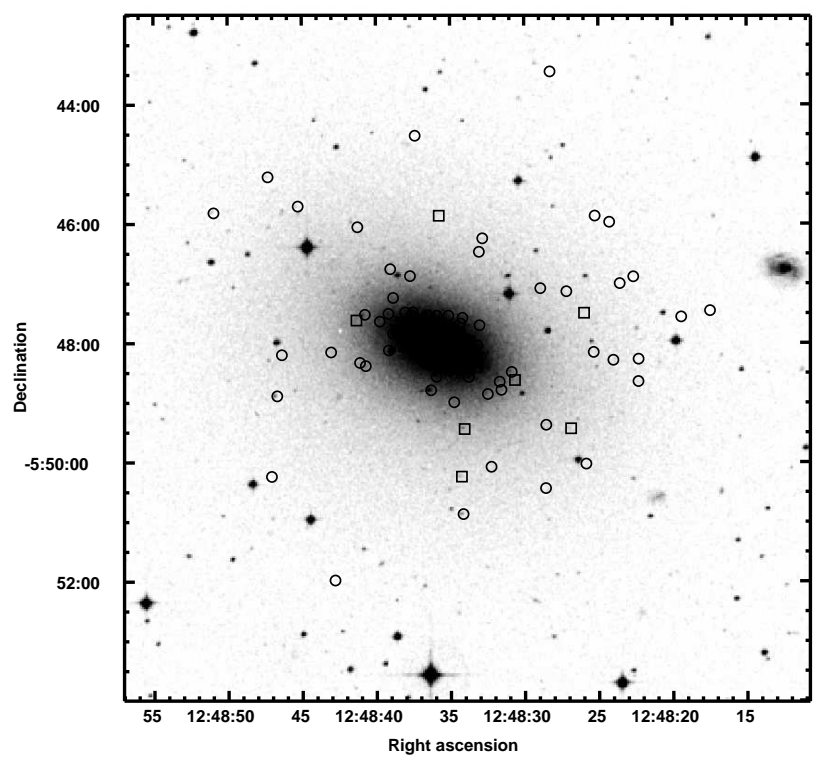

Fig. 8.5. Digital Sky Survey optical image of NGC 4697. The circles show the positions of the X-ray sources detected with Chandra. The squares indicate X-ray sources in known globular clusters. One should note that globular clusters have only been identified in this galaxy in an annulus from $1.5-2.5$ arcmin from the center. This figure was kindly provided by Craig Sarazin. It is adapted from Figure 3 of Sarazin, Irwin \& Bregman (2001).

$N G C 4472$ is a giant elliptical galaxy. In the inner regions of the galaxy it has been shown that metal-rich red globular clusters are about 3 times more likely to host a very luminous LMXB than the blue metal-poor ones (Figure 8.3). The X-ray luminosity does not depend significantly on the properties of the host globular cluster (Kundu et al. 2002).

$N G C 4365$, in the Virgo cluster, is one of a few early-type galaxies whose globular clusters do not have a bi-modal color distribution in $V-I$ (but it does in infrared colours, Puzia et al. 2002). Kundu et al. (2003) find that the presence of very luminous LMXBs is correlated with metallicity, but not with cluster age. The LMXB fraction per unit mass of the globular clusters is $\sim 10^{-7} M_{\odot}{ }^{-1}$. In contrast, Sivakoff et al. (2003) find that within the sample of IR-bright globular clusters studied by Puzia et al. (2002), the metal-rich, intermediate-age globular clusters, are four times as likely to contain LMXBs than the old globular clusters (with an uncertainty of a factor of two $)$. The luminosity function is a power-law with a cutoff at $\sim(0.9$ $2.0) \times 10^{39} \mathrm{erg} \mathrm{s}^{-1}$, much higher than the cutoff measured for other ellipticals.

$N G C 3115$ has a distinct bimodal color distribution of the globular clusters. The metal-poor blue and the metal-rich red globular clusters are both 12 Gyr old (Puzia et al. 2002). There are roughly equal numbers of red and blue globular clusters in 
the WFPC2 image. Kundu et al. (2003) find that the red globular clusters are the preferred sites for LMXB formation, largely as a consequence of their higher metallicity.

$N G C 140 \%$. White (2002) reported that about $90 \%$ of the 160 detected LMXBs have X-ray luminosities which exceed the Eddington limit for neutron stars. He suggests that many may be black hole binaries (rather than multiple neutron-star binaries within individual globular clusters), since $45 \%$ do not reside in globular clusters. To date (September 2004), these results have not yet been published in a refereed journal.

NGC 1553 is an S0 galaxy. 30\% of the emission in the $0.3-10 \mathrm{keV}$ band and $60 \%$ of the emission in the $2.0-10 \mathrm{keV}$ band is resolved into discrete sources (Blanton, Sarazin \& Irwin, 2001).

Kissler-Patig (1997) lists a global specific frequency of $2.3 \pm 0.5$, higher than the value listed in Table 8.3.

NGC 4649 (M60) is a bright elliptical galaxy. It was observed by Randall, Sarazin \& Irwin (2003); for details see Table 8.3.

NGC 1316 (Fornax A) is a disturbed elliptical radio galaxy with many tidal tails. Several mergers must have occurred over the past 2 Gyr (see Kim \& Fabbiano 2003, and references therein). One of the 5 globular cluster sources is super-soft. For an adopted distance of $18.6 \mathrm{Mpc}, 35 \%$ of the sources are above the Eddington limit of a $1.4 M_{\odot}$ neutron star (Kim \& Fabbiano, 2003). The luminosity function is well represented by a power law with a slope of -1.3 .

$N G C$ 720. 3 of the 12 globular cluster sources have X-ray luminosities in excess of $10^{39} \mathrm{erg} \mathrm{s}^{-1}$ (at $35 \mathrm{Mpc}$ ). It is possible that this galaxy is much closer, and that none of the sources are ultra-luminous (Jeltema et al. 2003).

$N G C 4486$ (M 87) is a giant elliptical galaxy near the center of the Virgo cluster, and has the most populous globular cluster system in the local supercluster. More luminous, redder and denser clusters are more likely to harbor a luminous X-ray source. Metal-rich red globular clusters are about three times more likely to host a luminous LMXB than the blue metal-poor ones; the trend with central density gives strong evidence that encounter rates are important in forming LMXBs in globular clusters. The trend with luminosity can arise as a consequence of the fact that more luminous clusters have higher encounter rates. The X-ray luminosity functions of both globular cluster-LMXBs and non-globular cluster LMXBs are well described by single power laws with an upper cutoff at $\sim 10^{39} \mathrm{erg} \mathrm{s}^{-1}$ (Jordán et al. 2004).

\subsubsection{Spiral Galaxies}

Plenty of spirals have been observed with Chandra, but there is very little information on the optical identifications. Globular clusters are hard to find because of the patchy extinction. There is also the difficulty of subtracting the diffuse light of the galaxy. These problems are exacerbated for nearly face-on spirals. In addition to the low-mass X-ray binaries, a spiral galaxy also hosts high-mass X-ray binaries and supernova remnants among the luminous X-ray sources.

M31 (Andromeda Nebula). The apparent size of M 31 is so big that only ROSAT has studied the whole (Magnier et al. 1992; Supper et al. 1997). Di Stefano et al. (2002) have conducted Chandra observations of $\sim 2560 \mathrm{arcmin}^{2}$ in four different areas 

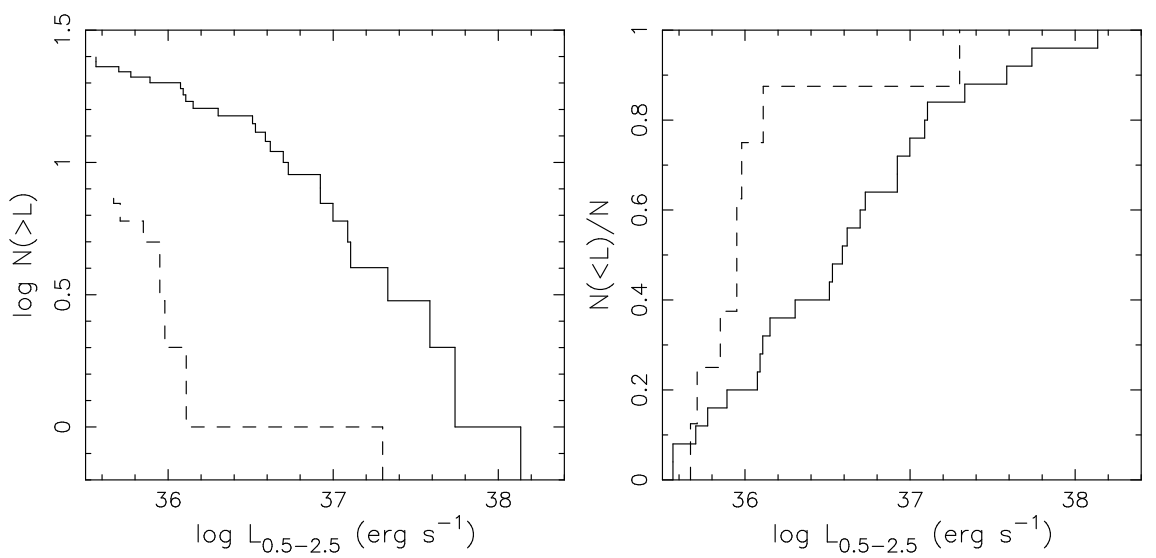

Fig. 8.6. Left: Comparison of the cumulative (from high luminosities downward) $X$-ray luminosity distributions of globular clusters in the Milky Way (dashed curve) and M 31 (solid curve). After Di Stefano et al. (2002). Right: Normalized cumulative (from low-luminosities upward) X-ray luminosity function for sources with $L_{x}>10^{35.5} \mathrm{erg} \mathrm{s}^{-1}$ in the Milky Way (dashed curve) and M 31 (solid curve). The Chandra luminosities given by Di Stefano et al. (2002) were multiplied by 0.46 to convert them to the energy range of the ROSAT data from Verbunt et al. (1995). The probability that the normalized distributions are the same is 0.03.

so as to be representative of the whole. About one third of the 90 Chandra sources have luminosities $(0.5-7 \mathrm{keV})$ in excess of $10^{37} \mathrm{erg} \mathrm{s}^{-1}$; the most luminous source is probably associated with the globular cluster Bo 375. Its luminosity (0.5-2.4 keV) varied between $\sim 2 \times 10^{38}$ and $\sim 5 \times 10^{38} \mathrm{erg} \mathrm{s}^{-1}$. Supper et al. (1997) reported regular variations of $\sim 50 \%$ on a timescale of $\sim 16$ hours. A similar percentage variability was found in the 500 day X-ray light curves of two other highly luminous globular clusters in M31, Bo 82 and Bo 86 (Di Stefano et al. 2002). Some of the more luminous globular cluster X-ray sources could be multiple sources.

It has been stated on the basis of different data sets that the X-ray luminosity function of globular cluster X-ray sources is different in M 31 than in the Milky Way (Van Speybroeck et al. 1979 on the basis of Einstein data; Di Stefano et al. 2002), and that it is the same (Supper et al. 1997). In Figure 8.6 we show the normalized cumulative distributions for clusters in the Milky Way and in M 31. The distributions look different, but a Kolmogorov-Smirnov test shows that there is a non-negligible probability, 0.03 , that the difference is due to chance. It is therefore possible that the extent to higher luminosities in M 31 is due to the larger number of X-ray sources (and of globular clusters).

M104 (NGC 4594, Sombrero galaxy) is an Sa galaxy at a distance of $\sim 8.9$ Mpc. Only optically bright globular clusters house the luminous LMXBs detected with Chandra (Di Stefano et al. 2003). The majority of the sources with luminosities in excess of $10^{38} \mathrm{erg} \mathrm{s}^{-1}$ are located in globular clusters. The luminosity function of X-ray sources in the globular clusters has a cut-off near the Eddington limit for a $1.4 M_{\odot}$ neutron star. One globular cluster houses a super-soft source (see $\S 11$ ). There is a connection between metal-rich, red globular clusters and the X-ray sources. 
However, the most luminous X-ray sources are equally likely to be located in metalpoor globular clusters with lower optical luminosities. The optically brightest blue globular clusters do not seem to house very luminous X-ray sources.

\subsubsection{NGC 5128 - Cen A}

This galaxy is probably the result of mergers, and consequently it is somewhat like a mixture between an elliptical and a spiral. Four X-ray sources outside the WFPC2 FOV are coincident with globular clusters (Kraft et al. 2001; Minniti et al. 2004). $70 \%$ of the globular cluster sources have luminosities in excess of $10^{37} \mathrm{erg} \mathrm{s}^{-1}$. There is no indication that any of them are black hole binaries. The globular cluster X-ray sources are preferentially found in massive globular clusters. Most of the globular clusters which harbor a luminous X-ray source have red colors (metal-rich). NGC 5128 is at a low galactic latitude; there is a lot of foreground extinction. This makes it difficult to get reliable optical data on globular clusters.

\subsubsection{Comparison and interpretation}

Many galaxies contain a substantially larger number of luminous X-ray sources in globular clusters than our own galaxy (compare Tables 8.1 and 8.3). This can be explained by their larger numbers of globular clusters. The fraction of globular clusters that contains a luminous X-ray source is roughly constant between galaxies, as is the number of X-ray sources in clusters scaled on cluster luminosity or mass $\left(2 \times 10^{-7} L_{\odot, I}{ }^{-1}\right.$ for $L_{x}>3 \times 10^{37} \mathrm{erg} \mathrm{s}^{-1}$, Sarazin et al. 2003, Kundu et al. 2003). Similarly, the larger number of globular cluster X-ray sources in M 31 compared to the Milky Way may be explained by the larger number of clusters (Supper et al. 1997, Di Stefano et al. 2003). Several authors reported a knee near the Eddington luminosity for an accreting neutron star in the luminosity functions of ellipticals (Sarazin et al. 2000, 2001 for NGC 4697; Kundu et al. 2002 for NGC 4472; Blanton et al. 2001 for NGC 1553, and Randall et al. 2003 for NGC 4649). However, Kim \& Fabbiano (2004), who corrected the Chandra data for incompleteness, have shown that the luminosity functions for each of the observed elliptical galaxies can be fit with one power law; two power laws do not improve the fit in a significant way. It is interesting to note that even though no breaks in the individual luminosity functions are significant, if the luminosity functions of all observed ellipticals (containing a total of about 985 point-like sources) are added, a broken power-law fit is a better fit than a single power law; the break is near $5 \times 10^{38} \mathrm{erg} \mathrm{s}^{-1}$ (Kim \& Fabbiano, 2004; $\S 12.4 .3)$.

Clearly, a large number of LMXBs have luminosities substantially above the Eddington luminosity of an accreting neutron star. In analogy with the luminosity distribution in the Milky Way (Grimm et al. 2002), this suggests that many of these sources may be accreting black holes. This suggestion is supported in some cases by the X-ray spectrum, which shows the soft signature of an accreting black hole (e.g. Angelini et al. 2001). The fact that a very luminous accreting black hole is not found in the globular clusters of the Milky Way is probably due to the small number of cluster sources.

An alternative explanation for the LMXBs with luminosities substantially above the Eddington luminosity of an accreting neutron star is provided by Bildsten \& 

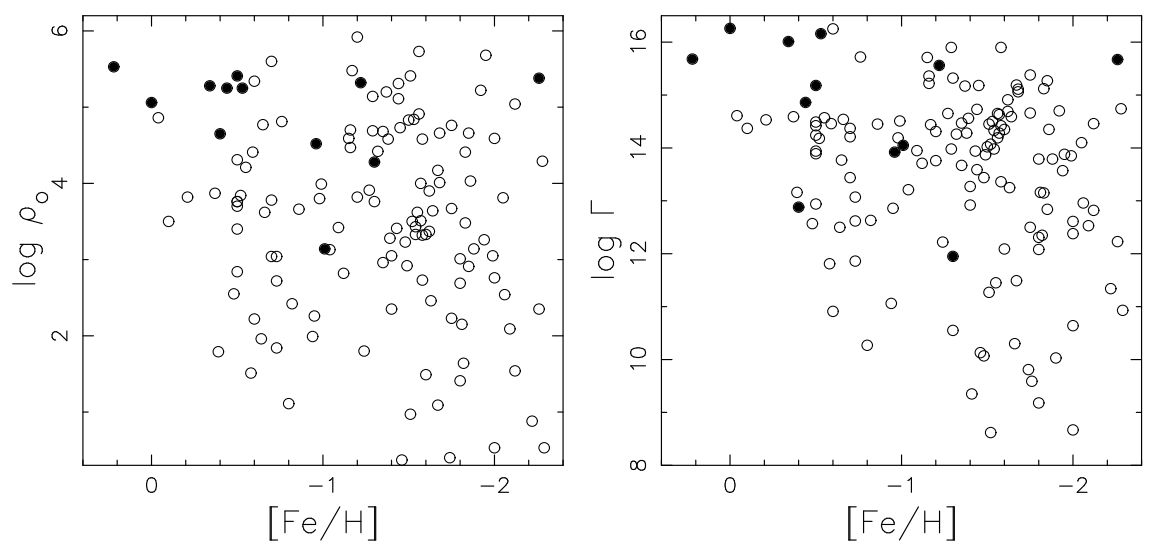

Fig. 8.7. Left: central density of globular clusters in the Milky Way as a function of metallicity. Filled circles indicate globular clusters with a luminous X-ray source. Even at the same density there is a preference for high-metallicity clusters. After Bellazzini et al. (1995). Right: the preference for high-metallicity clusters persists in a plot of collision number (Eqs. 8.5 and 8.6) as a function of metallicity.

Deloye (2004), who note that the Eddington limit for hydrogen-poor gas is higher. They show that the high luminosities can be explained by invoking binaries in which a helium or carbon/oxygen white dwarf of 0.04-0.08 $M_{\odot}$ transfers mass to a neutron star, at orbital periods of 5-10 minutes. From the evolution of such binaries, driven by gravitational radiation, to longer periods and lower mass-transfer rates the expected luminosity function can be computed, and is found to be compatible with the observed luminosity function.

The X-ray sources are found preferably in optically bright clusters (Angelini et al. 2001). This could be explained as a scaling with mass (Kundu et al. 2002, Sarazin et al. 2003). We suggest, however, that the scaling with mass is a proxy for the scaling with the collision number, caused by the strong correlation between mass and collision number. In the Milky Way, the probability of a cluster to contain a luminous X-ray source scales better with the collision number than with the mass (Verbunt \& Hut 1987; Pooley et al. 2003).

In many galaxies, luminous X-ray sources are found preferably in red, metalrich clusters. Bellazzini et al. (1995) demonstrated this for the Milky Way (see Figure 8.7) and less conclusively for M31. Di Stefano et al. (2003) find in their sample of M 31 clusters that the probability that a cluster contains an X-ray source is not strongly correlated with metallicity. Kundu et al. (2002) find that a red cluster in NGC 4472 has a 3 times higher probability of hosting a luminous X-ray source than a blue cluster. A similar result is found for NGC 4365 by Sarazin et al. (2003), and for NGC 3115 by Kundu et al. (2003). We consider four suggested explanations. First, if metal-rich clusters are younger, they contain main-sequence stars of higher mass, which are thought to be more efficient in forming an X-ray binary (Davies \& Hansen 1998). In NGC 4365 such a young population is indeed present, but it does not show an increased formation rate of X-ray sources (Kundu et al. 2003). Also, the preference for metal-rich clusters is observed in the Milky 
Way and in NGC 3115, where all globular clusters are old. These results show that metallicity, not age, must explain the preference of X-ray sources for red clusters (Kundu et al. 2003). Second, a higher X-ray luminosity at higher metallicity would produce a preference for metal-rich clusters in a flux-limited sample. Various models have been suggested to produce higher X-ray luminosities in binaries with a donor of higher metallicity (e.g. Bellazzini et al. 1995, Maccarone et al. 2004). However, $\mathrm{X}$-ray sources in metal-rich clusters are not observed to be more luminous than those in metal-poor clusters in M 31 (Verbunt et al. 1984) or, with less statistical constraint, in NGC 4472 (Maccarone et al. 2003). Third, Grindlay (1987) suggests that metal-rich clusters have a flatter initial mass function (and hence more neutron stars). However, such a dependence is not observed in the Milky Way (Piotto \& Zoccali 1999). Finally, Bellazzini et al. (1995) suggest that the longer life times and larger radii of metal-rich stars enhance their capture rate; the capture probability is proportional to radius (see Eq.8.5 below), and it must be doubted that the small difference in radii has sufficient effect to explain the observations (Maccarone et al. 2004). It is fair to say that the connection between metallicity and the occurrence of LMXBs in globular clusters is not yet well understood.

There is a tendency for X-ray sources in metal-rich globular clusters to have softer X-ray spectra (M 31: Irwin \& Bregman 1999, NGC 4472: Maccarone et al. 2003).

\subsubsection{Comparison between field and cluster sources}

The X-ray luminosity function of sources in globular clusters is not very different from that of the sources outside globular clusters (Maccarone et al. 2003, Sarazin et al. 2003). The spatial distribution of X-ray sources outside globular clusters in elliptical galaxies is similar to that of the globular cluster sources. In elliptical galaxies, globular clusters often harbor a very large fraction of all X-ray sources (Table 8.3). This has raised the suggestion that all X-ray sources in elliptical galaxies originate in globular clusters (White et al. 2002). The field sources then could have been ejected from a cluster, or originate in a cluster that was later destroyed by the galactic tidal field. The demand that a cluster lives long enough to form X-ray binaries, and short enough not to be around now, requires fine tuning. Thus, the ejection hypothesis may be more probable.

This would suggest that a large number of globular clusters translates into a large number of X-ray sources, both in the clusters and (due to ejection) outside them. The fraction of X-ray sources in globular clusters would then be similar for different galaxies. In the Milky Way and in M 31 there are about 10 luminous low-mass X-ray binaries in the disk for each one in a globular cluster. In elliptical galaxies, there is of order 1 low-mass X-ray binary outside clusters for each one in them (see Table 8.3). This indicates that the majority of the disk sources in the Milky Way and M31, and by extension in spiral galaxies in general, are formed in the disk; although as noted in the Introduction some individual systems may have escaped from globular clusters. [One should note that the HST field of view is much smaller than that of Chandra. Therefore, in comparing the number of X-ray sources associated with globular clusters with those not located in globular clusters (Table 8.3), in all those cases where HST data were needed to identify the clusters, one can only consider the X-ray sources which are detected in the regions observed with HST.] 
For elliptical galaxies the case is less clear. Using optical luminosities of the galaxies and the specific globular cluster frequencies, White, Sarazin \& Kulkarni (2002)

reported that they found evidence that the sum of the X-ray luminosities of all X-ray sources in ellipticals scales approximately with the number of globular clusters, and they conclude that this indicates that the population outside clusters is formed in the clusters. However, the uncertainties in the specific frequencies may be substantially larger than the values used by these authors, and that makes it difficult to quantify their findings. Kim and Fabbiano (2004) have made a similar study, and caution about the above interpretation.

The fraction of low-mass X-ray binaries in clusters ranges from about 20 to $70 \%$ in ellipticals (see Table 8.3). This suggests, in our opinion, that globular clusters alone are not responsible for all low-mass X-ray binaries. In systems with small numbers, the total luminosity can be affected by just a couple of very luminous sources; the number of sources may therefore be a better estimator for the population size than the integrated X-ray luminosity. Clearly, the origin of low-mass X-ray binaries in elliptical galaxies deserves more study.

If the majority of those luminous LMXBs in elliptical galaxies not located in globular clusters are primordial, their luminosities could not have been constant throughout their lifetimes (because the product of age and the required mass-transfer rate would exceed the donor mass). There are two ways out of this lifetime problem: (i) they are not primordial but they were formed in globular clusters, and somehow released into the field, or (ii) the majority of them are transients with a low duty cycle (see Piro \& Bildsten 2002). If the latter is the case, follow-up observations with Chandra will be able to reveal the variability if a sufficient number of them have outbursts that last only a few years and not much longer. We may add a third solution, which is that (iii) systems formed from primordial binaries will emerge from their early evolution as neutron stars or black holes with detached main-sequence companions. How long it takes for the binary to turn into an X-ray source then depends on the time required for the orbit to shrink due to loss of angular momentum, or for the donor to expand into a giant after completing its main-sequence evolution (for reviews see Verbunt 1993 and Chapter 16 by Tauris and Van den Heuvel). It may be noted that binaries formed in a globular cluster may also go through a long-lived detached phase (Grindlay 1988).

\subsection{Low-luminosity X-ray sources}

As already mentioned, a limited number of low-luminosity sources has been detected with Chandra in several clusters which contain a luminous X-ray source. The presence of such a source limits the sensitivity with which low-luminosity sources can be detected, because of the wings of the point spread function. The sharp $\left(<1^{\prime \prime}\right)$ images and high sensitivity of the Chandra observations is best used in clusters which do not contain a luminous source. Such observations show that the central regions of several globular clusters contain dozens of sources. As a typical example, the distribution of the sources in NGC 6440 is concentrated towards the cluster center; while it spreads beyond the core radius, it is fully contained within the half-mass radius (Figure 8.8). From this spatial distribution alone, it can be safely asserted that almost all sources detected are related to the globular cluster. In clusters with large 


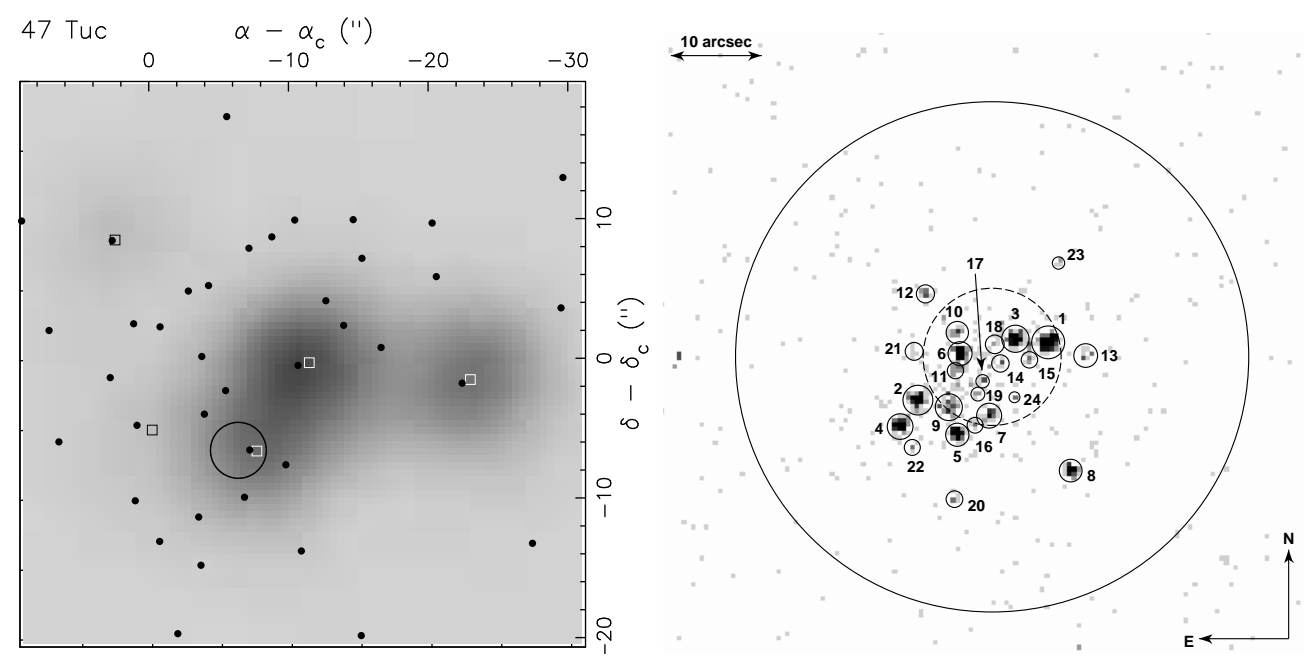

Fig. 8.8. Left: The impact of Chandra on the study of low-luminosity X-ray sources in globular clusters is well illustrated by the observations of 47 Tuc. The grey scale of the smoothed ROSAT-HRI countrate indicates the resolution obtained with this instrument (Verbunt $\mathscr{E}$ Hasinger 1998). The circle indicates the position (1 $\sigma$ region) of the single Einstein source (Hertz \& Grindlay 1983), squares indicate the positions of the five ROSAT sources in this region, filled circles are 39 Chandra positions (Grindlay et al. 2001a). Right: Projected distribution of X-ray sources in the globular cluster NGC 6440. The dashed and solid lines indicate the core and half-mass radii, respectively. From Pooley et al. (2002b). In the case of 47 Tuc, each ROSAT source corresponds to one Chandra source; in the case of NGC6440, two sources previously found by ROSAT are both resolved into multiple sources.

apparent core radii and/or half-mass radii, a large fraction of the detected sources may be fore- or background sources; an example is $\omega$ Centauri.

In trying to determine the nature of all these X-ray sources, we may be guided by our knowledge from previous satellites, in particular ROSAT. Such guidance allows us to make a preliminary classification of a source based on its X-ray flux and spectrum. If a secure optical counterpart is found - which thanks to the accurate source positions of Chandra is often the case whenever sufficiently deep HST observations are available - the classification of a source can be further based on its optical spectrum, and on the ratio of the X-ray and optical fluxes. A secure classification can also be found if the position of a radio pulsar coincides with that of an X-ray source: radio and X-ray positions are so accurate that the probability of a chance coincidence is virtually negligible for these rare objects.

Our discussion of the low-luminosity sources proceeds through the various classes illustrated in Figure 8.1 viz. low-luminosity low-mass X-ray binaries, recycled radio pulsars, cataclysmic variables, and magnetically active close binaries. An overview of published Chandra observations of low-luminosity sources in globular clusters is given in Table 8.4 


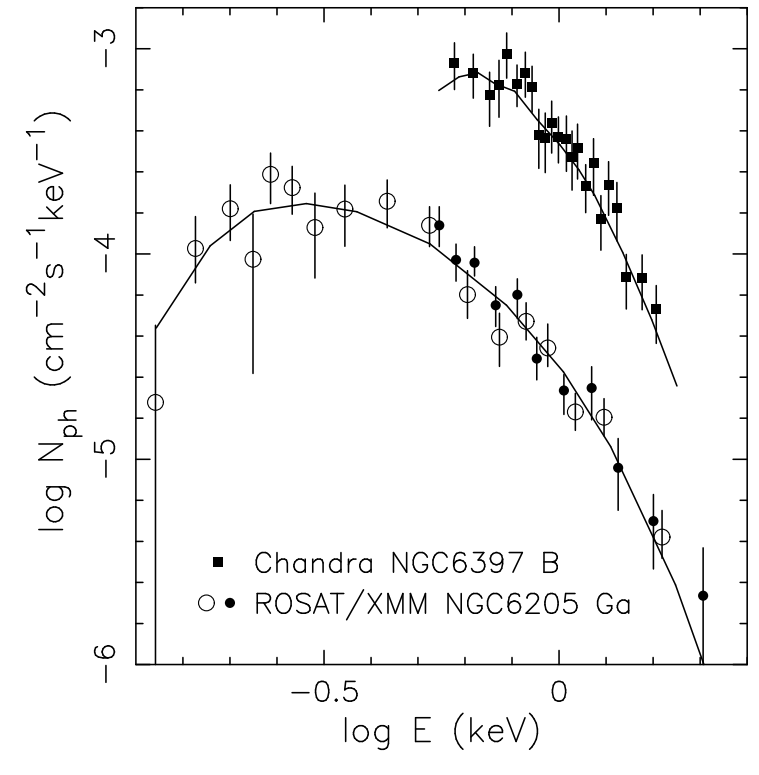

Fig. 8.9. X-ray spectra of lowluminosity $X$-ray binaries with neutron stars, as observed (i.e., not corrected for interstellar absorption) with ROSAT and $X M M$ for the source $G a$ in NGC 6205 (M 28) (Gendre et al. 2003b; Verbunt 2001); and with Chandra for the source $B$ in NGC 6397 (shifted upwards by 1 decade; in 't Zand, private communication; Grindlay et al. 2001b). The solid lines show fits with models for hydrogen atmospheres of neutron stars.

\subsubsection{Low-luminosity low-mass $X$-ray binaries}

We consider a low-luminosity low-mass X-ray binary with a neutron star, $\operatorname{LMXB}^{\mathrm{NS}}$, securely classified when its luminosity is high enough $\left(L_{\mathrm{X}} \gtrsim 10^{32} \mathrm{erg} \mathrm{s}^{-1}\right)$ and its X-ray spectrum is soft (black body color temperature about 0.1 to $0.3 \mathrm{keV}$ ). The reason for this is that most soft X-ray transients in the galactic disk have these properties when they contain a neutron star. Their quiescent X-ray spectra have been roughly described as Planck spectra with a temperature of about $0.3 \mathrm{keV}$ (Verbunt et al. 1994), but more correctly should be fitted with model spectra of neutron star atmospheres as have been computed by e.g. Rajagopal \& Romani (1996) and Zavlin et al. (1996). For quiescent transients in the disk, such fits give effective temperatures of $0.1-0.2 \mathrm{keV}$ and neutron star radii of roughly $10 \mathrm{~km}$ (Rutledge et al. 1999). The situation is more problematic if a transient in quiescence has a power-law spectrum and a luminosity in the range $10^{31}-10^{34} \mathrm{erg} \mathrm{s}^{-1}$. In that case, the system could be either a $\mathrm{LMXB}^{\mathrm{N} S}$ or a low-mass X-ray binary with a black hole, $\mathrm{LMXB}^{\mathrm{B} H}$ (see Tomsick et al. 2003, Wijnands et al. 2005). A hard spectrum can also indicate a cataclysmic variable, as may be the case for one or two sources in NGC 6652 and Terzan 1.

Chandra and XMM are sensitive enough to detect luminosities of $L_{x} \gtrsim 10^{32} \mathrm{ergs}^{-1}$ in any cluster that they observe, with sufficient counts to determine whether the spectra are power laws or thermal (i.e., soft). Sources for which fits with neutron star atmosphere models have been shown to give a good description of the X-ray spectrum include X7 in $\omega$ Cen (Rutledge et al. 2002, see also Gendre et al. 2003a), X5 and X7 in 47 Tuc (Heinke et al. 2003b), B in NGC 6397 (Grindlay et al. 2001b), CX1 in NGC 6440 (in 't Zand et al. 2001), and Ga in NGC 6205 (M 13, Gendre et al. 2003b). Most of these sources were already detected with ROSAT, being (among) the most luminous sources in each cluster (the exception is CX1 in NGC 6440). As 
noted above, CX1 in NGC 6440 is the transient, detected in the bright state in 1998 and 2001; whether the transient of 1971 was the same source cannot be ascertained. This source supports our premiss that the more luminous $\left(L_{x} \gtrsim 10^{32} \mathrm{erg} \mathrm{s}^{-1}\right)$ among the low-luminosity soft sources are quiescent accreting neutron stars.

Probable classifications as low-luminosity $\mathrm{LMXB}^{\mathrm{N} S}$, based on the ratio of soft to hard counts as detected with Chandra have been suggested for 4 of the most luminous faint sources in NGC 6440 (Pooley et al. 2002b), and in Terzan 5 (Heinke et al. 2003a). Further probable identifications are based on the luminosity of the sources: 3 low-luminosity $\mathrm{LMXB}^{\mathrm{NS}}$ (in addition to the Rapid Burster) in Liller 1 (Homer et al. 2001b), 1 or 2 in NGC 6652 (Heinke et al. 2001). We want to point out, however, that it cannot be excluded that some of these are black-hole binaries.

A low-mass X-ray binary with a black hole can have a much lower luminosity than a $\mathrm{LMXB}^{\mathrm{NS}}$; as an example, for the transient A0620-00 in quiescence $L_{\mathrm{X}} \simeq$ $10^{30} \mathrm{erg} \mathrm{s}^{-1}$, much of which could even be due to the donor in the binary (Verbunt 1996, Bildsten \& Rutledge 2000). At such low luminosities, even Chandra or XMM observations cannot provide a secure classification, and consequently we have no information on the number of low-luminosity low-mass X-ray binaries with a blackhole accretor.

So far, only two low-luminosity $\mathrm{LMXB}^{\mathrm{N} S} \mathrm{~S}$ in globular clusters have been identified optically, one in 47 Tuc and one in $\omega$ Cen (Edmonds et al. 2002b, Haggard et al. 2004).

\subsubsection{Millisecond pulsars}

Most identifications of X-ray sources in globular clusters with recycled radio pulsars are based on positional coincidence. The exceptions are the identifications of the pulsar in NGC 6626 (M 28), which is based on the pulse period, and of pulsars in NGC 6397 (XB) and in 47 Tuc (W29/PSR W) which are based on their orbital periods.

The pulsar in M 28 is the only one in a globular cluster which was identified with an X-ray source before the Chandra observations. By comparing the on-pulse X-rays with the off-pulse X-rays, the X-ray spectrum of the pulse could be isolated (Saito et al. 1997). Chandra resolves the pulsar from other cluster sources and obtains a phase-averaged power law spectrum with photon index 1.2 (Becker et al. 2003).

Because accurate (timing) positions are not yet available for many of the radio pulsars, it is likely that some of them have been detected in X-rays already but not yet identified as such. In fact, an X-ray source in NGC 6397 was first identified with a possible BY Dra binary (Grindlay et al. 2001b); it was then found that this binary houses a radio pulsar (Ferraro et al. 2001). Similarly, NGC 6752 CX11 was identified by Pooley et al. (2002a) with a possible cataclysmic variable or background galaxy, but now is more probably identified with PSR D in that cluster on the basis of newly determined timing positions (D'Amico et al. 2002); positions of X-ray sources are coincident with the timing positions of PSRs C and (marginally) $\mathrm{B}$.

Verbunt et al. (1996) showed that for the radio pulsars detected in X-rays with ROSAT, $L_{0.1-2.4 \mathrm{keV}} \lesssim 10^{-3} L_{s d}$, where $L_{s d} \equiv I \Omega \dot{\Omega}$ is the loss of rotation energy, usually referred to as the spin-down luminosity, with $I$ the moment of inertia and $\Omega \equiv 2 \pi / P$. In accordance with this scaling, the radio pulsars detected in X-rays so far are those with the highest $L_{s d}$ of those in the clusters observed with Chandra. 
Fig. 8.10. X-ray luminosities as a function of spindown luminosities, $L_{s d}$, of radio pulsars in globular

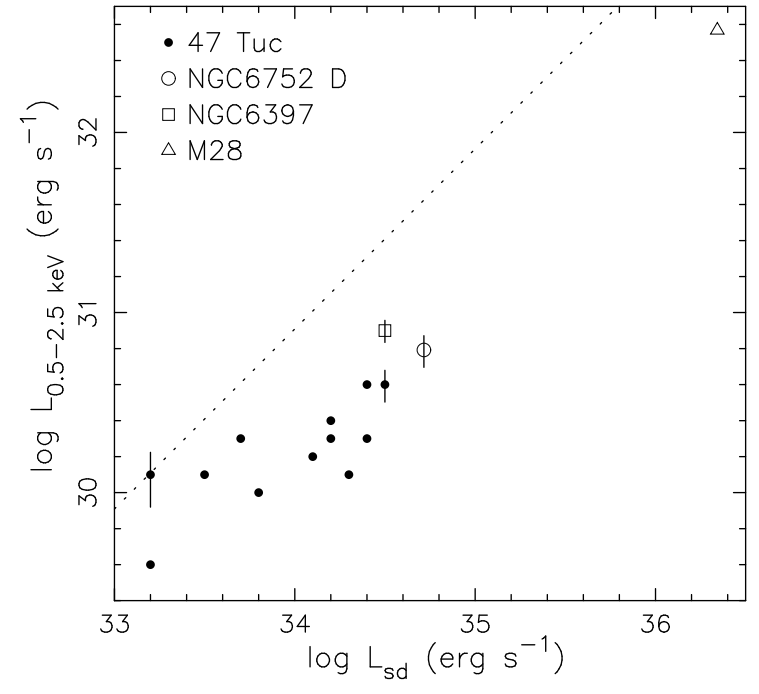

clusters. The unresolved pulsar pairs $G / I$ and $F / S$ in 47 Tuc have been omitted. The dotted line indicates $L_{0.1-2.4 \mathrm{keV}}=10^{-3} L_{s d}$ (Verbunt et al. 1996), with a small correction for the different $X$-ray energy range. The cluster pulsars lie below, but roughly parallel to this relation. Notice M28 in the upper right hand corner. Several $1 \sigma$ errors are shown; these are computed from Poisson statistics of the detected number of X-ray counts, and do not take into account uncertainties in the spectral energy distribution and/or cluster distance. Data from Grindlay et al. (2002), D'Amico et al. (2002), Becker et al. (2003) and Possenti et al. (quoted in Bassa 83 Stappers 2004).

Grindlay et al. (2002) assume that the electron density in 47 Tuc is homogeneous, and from small differences in dispersion measures determine the position of each pulsar along the line of sight; this is then used to correct the observed period derivative for gravitational acceleration in the cluster potential. Comparison of the corrected spindown luminosities with the X-ray luminosities led Grindlay et al. (2002) to suggest that $L_{x} \propto \sqrt{L_{s d}}$. They further note that the pulsar in NGC 6397 agrees with this (slower) trend, whereas the pulsar in M 28 does not. Grindlay et al. argue that the emission of the pulsar in M 28 is mainly magnetospheric in origin, whereas the emission of the other pulsars in globular clusters is mainly thermal emission from the surface of the neutron star.

We reinvestigated the relation between X-ray and the spindown luminosities for the globular cluster pulsars in Figure8.10 We include NGC 6752 D, and the pulsar in M 28. It should be noted that the luminosity of the pulsar in M 28 is only about $20 \%$ of the total cluster luminosity as observed with ROSAT (Verbunt 2001; Becker et al. 2003), whereas the value used by Grindlay et al. (2002) is the total cluster luminosity. Since thermal emission from millisecond pulsars is the result of heating by magnetospheric processes, we prefer not to exclude the magnetospheric X-rays and to retain the pulsar in $\mathrm{M} 28$, and we are inclined to conclude that the general slope of the relation between $L_{x}$ and $L_{s d}$ is similar to that observed for the pulsars detected in the Galactic disk, with some scatter at the lowest luminosities. The strong downward revision of the spindown luminosity of the pulsar in NGC6397 (Possenti et al. quoted in Bassa \& Stappers 2004) brings this pulsar also in line with the steeper dependence of $L_{x}$ on $L_{s d}$. 

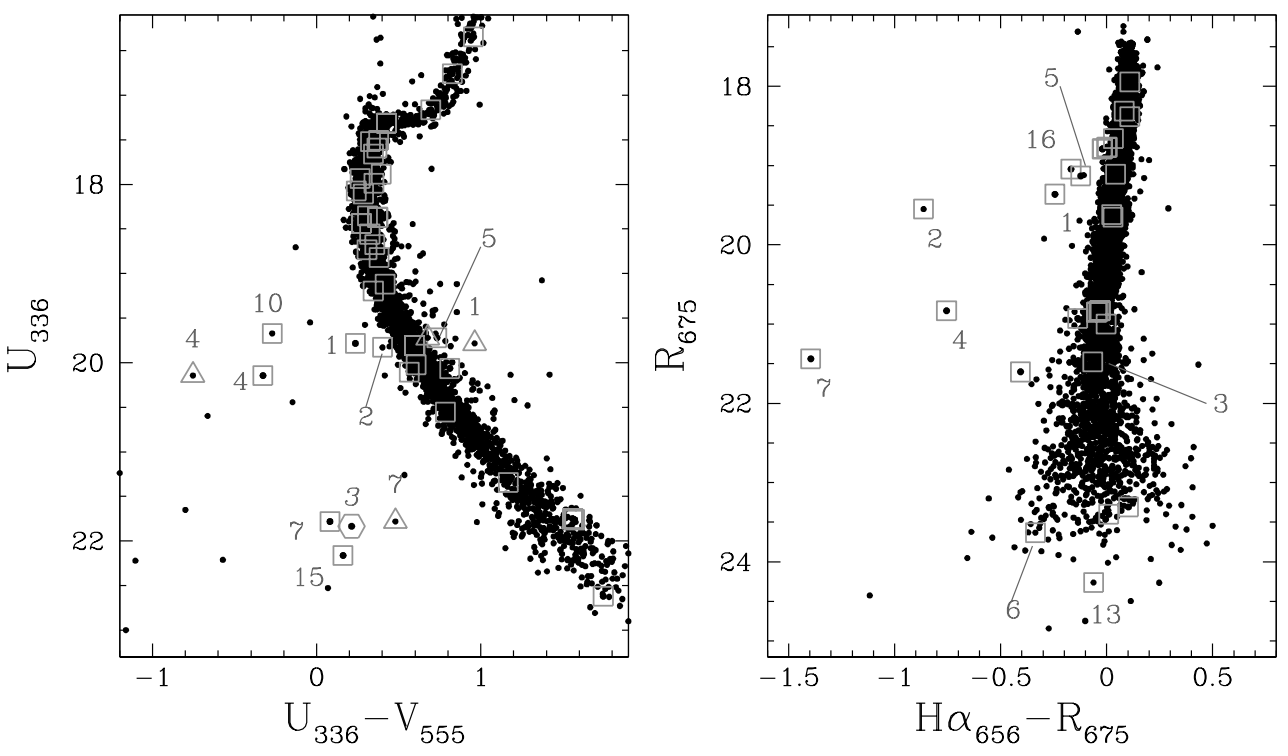

Fig. 8.11. $U-V$ and $H \alpha-R$ color magnitude diagrams of the central regions of NGC 6752. Stars within error circles of Chandra X-ray sources are indicated with squares; numbers indicate the corresponding Chandra source. Cataclysmic variables lie to the left of the main sequence in the $U-V$ diagram, i.e. they are blue.

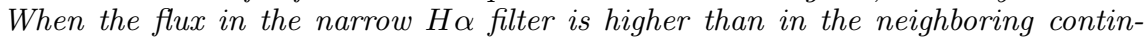
uum (measured in $R$ ), the points fall to the left of the main sequence in the $H \alpha-R$ diagram. Because of variability, the same object may lie in different locations of the color magnitude diagrams, depending on which data set is used. Updated after Pooley et al. (2002a).

\subsubsection{Cataclysmic variables}

Cataclysmic variables are best identified when an optical counterpart is found. A good indicator is that the optical counterpart is bluer than the main sequence, especially in the ultraviolet; and/or that it has strong $H \alpha$ emission (see Figure 8.11). As an example, such counterparts were identified in NGC 6397, and follow-up spectra show the strong Balmer emission lines prevalent in cataclysmic variables (Cool et al. 1995, Grindlay et al. 1995, Edmonds et al. 1999; note that firm identifications were only possible once Chandra had obtained accurate positions, Grindlay et al. 2001b). Quiescent neutron-star low-mass X-ray binaries also have blue spectra with Balmer emission, but can be distinguished from cataclysmic variables through their soft X-ray spectra, and by the fact that they are more luminuos than cataclysmic variables (see 88.4.1). Optical and ultraviolet color-magnitude diagrams have been used to classify optical counterparts as cataclysmic variables also in NGC 6752 and in 47 Tuc (Pooley et al. 2002a, Edmonds et al. 2003).

If no optical colors are available, the ratio of X-ray to optical flux provides a good, but not conclusive, indication as to whether a source is a cataclysmic variable, as shown with cataclysmic variables studied in the ROSAT All Sky Survey (Verbunt et al. 1997, Verbunt \& Johnston 2000). In Figure8.12 we show (a measure of) the X-ray 


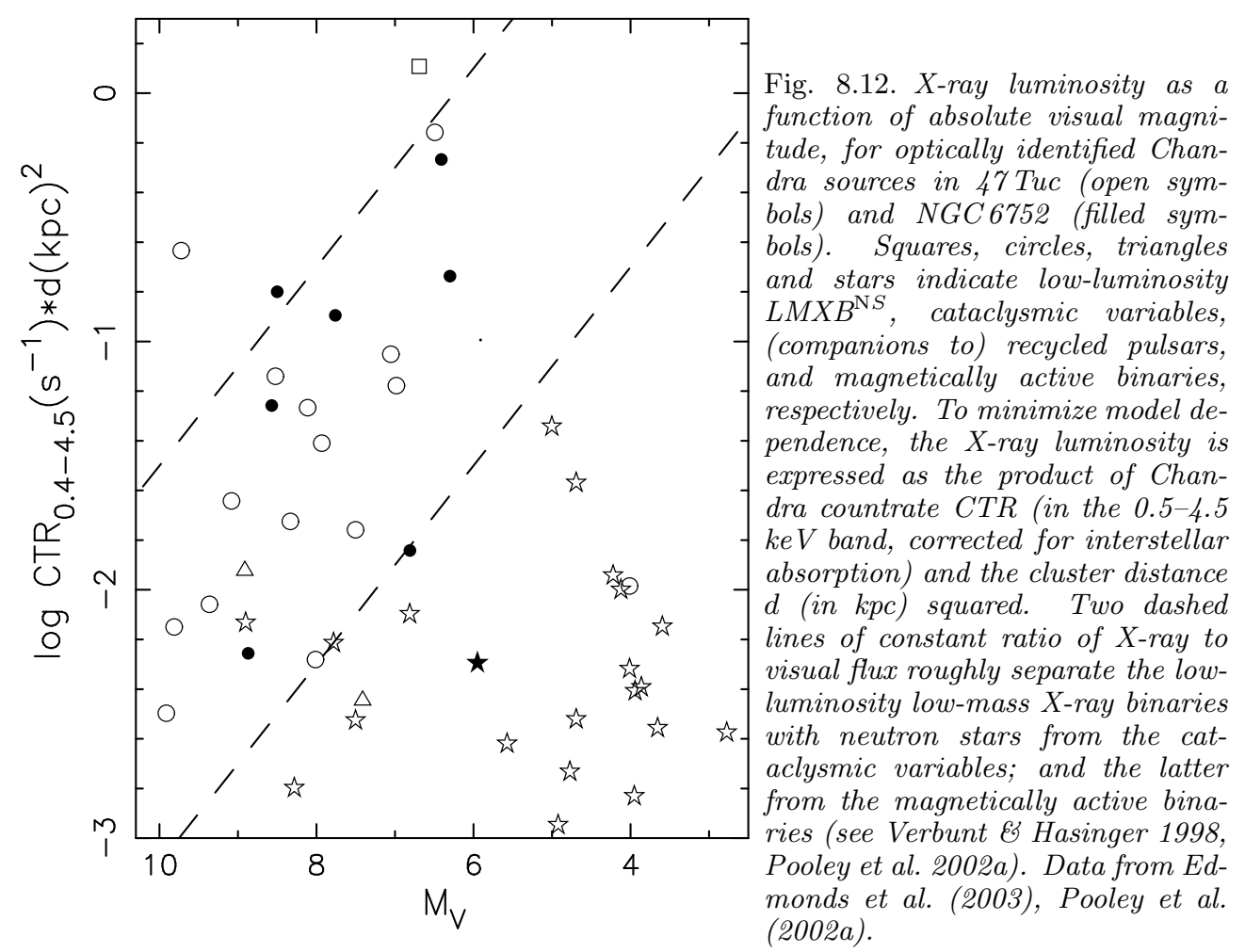

luminosity in the $0.5-4.5 \mathrm{keV}$ range as a function of the absolute visual magnitude for X-ray sources in 47 Tuc and in NGC 6752. Only sources which have been classified on the basis of optical/ultraviolet color magnitude diagrams are shown. In the figure we plot the line

$$
\log \left(\mathrm{CTR}_{0.5-4.5 \mathrm{keV}} d_{\mathrm{kpc}}{ }^{2}\right)=-0.4 M_{V}+0.9
$$

where $\mathrm{CTR}_{0.5-4.5 \mathrm{keV}}$ is the number of counts per second in the $0.5-4.5 \mathrm{keV}$ range, and $d_{\mathrm{kpc}}$ the distance in $\mathrm{kpc}$. This line roughly separates the cataclysmic variables from magnetically active binaries. A parallel line for an X-ray luminosity which is a factor $\simeq 40$ higher roughly separates the cataclysmic variables from the lowluminosity low-mass X-ray binaries with a neutron star. The figure shows that the ratio of X-ray to optical luminosity is a fairly good classifier of X-ray sources in the absence of more conclusive information.

A further indicator that a source is a cataclysmic variable may be found from optical variability, either orbital or from a (dwarf) nova outburst. Orbital variability may be present in magnetically active binaries too, and thus can be used to classify a source only in combination with other information, such as color magnitude diagrams, or ratio of X-ray to visual flux. Two cataclysmic variables were found in NGC 6752 based on periodic variability and $H \alpha$ emission by Bailyn et al. (1996), and were identified with Chandra X-ray sources by Pooley et al. (2002a). Variability indicative of dwarf nova outbursts has been detected for several blue objects in 
47 Tuc (e.g. Paresce et al. 1992, Paresce \& De Marchi 1994, Shara et al. 1996); these sources have subsequently been identified with Chandra X-ray sources (Grindlay et al. 2001a). An optical variable in the core of NGC 6656/M 22 has been identified as a possible dwarf nova, detected in X-rays with Einstein, ROSAT and XMM (Anderson et al. 2003; see Table8.2).

So far, only 47 Tuc, NGC 6397 and NGC 6752 have been studied to such an extent that a large fraction of the X-ray sources in them has been optically identified. Most of them are classified as cataclysmic variables. In $\omega$ Cen, several Chandra sources have been identified with (optically detected) cataclysmic variables (Carson et al. 2000), but HST observations only cover a small fraction of the cluster. Classifications based only on the X-ray to optical flux ratio must be considered preliminary, as illustrated by the case of NGC 6752 CX11 (see 88.4.2).

In general it may be stated that the properties of cataclysmic variables in globular clusters are similar to those of cataclysmic variables in the Galactic disk (i.e. in the solar neighborhood; see also §10). In the Galactic disk, distances and interstellar absorption for cataclysmic variables are only inaccurately determined at best. In contrast, for systems in globular clusters these quantities may be set equal to the values for the cluster, which are much better known. Thus comparison between different classes of objects will be more accurate in globular clusters.

As an example, we note that Verbunt \& Hasinger (1998) in their analysis of ROSAT observations of 47 Tuc use the ratio of X-ray to visual flux to suggest that 47 Tuc $\mathrm{X}$ 9, identified with the blue variable V1, is a low-luminosity low-mass X-ray binary with a neutron star. In Figure 8.12 based on more accurate Chandra data and now secure identifications, the systems with the three highest X-ray to optical flux ratios in 47 Tuc are X10/V3, X7 and X9/V1. X7 is indeed a low-luminosity low-mass Xray binary with a neutron star, but the hard X-ray spectra of X10 and X9 indicate that they are probably cataclysmic variables. This illustrates the overlap between

low-mass X-ray binaries and cataclysmic variables in the X-ray to visual flux ratio.

\subsubsection{Magnetically active binaries}

$\mathrm{X}$-ray sources in globular clusters can be classified as magnetically active binaries when a stellar flare is observed in X-rays; or on the basis of the optical counterpart, when this is a known active binary, or less securely when it lies above the main sequence and/or shows weak $H \alpha$ emission.

Two OGLE variables in NGC 5139, OGLEGC15 and OGLEGC22, are identified by Cool et al. (2002) with Chandra sources (not listed by Rutledge et al. 2002, but confirmed by Gendre, private communication). A third OGLE variable in NGC 5139, OGLEGC30, has been detected with XMM (Gendre et al. 2003a). Yet another Chandra X-ray source, already detected with ROSAT but not detected with XMM and therefore a variable $\mathrm{X}$-ray source, shows $H \alpha$ emission, and presumably is also a magnetically active binary (Gendre et al. 2003a). Figure 8.12 shows Chandra X-ray sources in 47 Tuc and NGC 6752 that are classified on the basis of color-magnitude diagrams as magnetically active binaries; for many of these binaries in 47 Tuc the orbital lightcurve confirms their identity as coronal X-ray emitters (Edmonds et al. 2003). That care must be taken in classifying sources is shown by the example of NGC 6397 CX12 (see 8.4 .2 ). 


\begin{tabular}{|c|c|c|c|c|c|c|c|c|c|}
\hline cluster & ref & $L_{\text {low }}$ & $\mathrm{BX}$ & FX & $\mathrm{CV}$ & PSX & (PSR) & BY & $N_{t o t}$ \\
\hline NGC 6440 & 180 & $2 \times 10^{31}$ & 1 & 3 & & & & & \\
\hline NGC 6652 & 92 & $8 \times 10^{32}$ & 1 & 3 & $\rightarrow 1$ ? & & & & \\
\hline Terzan 1 & 243 & $3 \times 10^{33}$ & 1 & 1 & $\rightarrow 1 ?$ & & & & \\
\hline Terzan 5 & 93 & $5 \times 10^{32}$ & 1 & 4 & 5 & & $(4)$ & & \\
\hline Liller 1 & 108 & $\sim 10^{34}$ & 1 & 3 & & & & & \\
\hline 47 Tuc & 81 & $10^{30}$ & 0 & 2 & $>30$ & 15 & $(22)$ & 26 & 104 \\
\hline$\omega$ Cen & 65 & $10^{31}$ & 0 & 1 & $>20$ & & $(0)$ & 4 & $\sim 100$ \\
\hline NGC 6093 & 95 & $7 \times 10^{30}$ & 0 & 2 & $\sim 15$ & & & & 19 \\
\hline NGC 6121 & 14 & $1 \times 10^{29}$ & 0 & 0 & 3 & 1 & (1) & 14 & $\sim 20$ \\
\hline NGC 6205 & 66 & $2 \times 10^{31}$ & 0 & 1 & 4 & 0 & (5) & & 5 \\
\hline NGC 6397 & 82 & $3 \times 10^{29}$ & 0 & 1 & 9 & 1 & (1) & 3 & $\sim 20$ \\
\hline NGC 6626 & 15 & $2 \times 10^{30}$ & 0 & 1 & $\sim 25$ & 1 & (1) & & \\
\hline NGC 6656 & 237 & & 0 & $1 ? \leftarrow$ & 3 & & & $\sim 3$ & \\
\hline NGC 6752 & 179 & $2 \times 10^{30}$ & 0 & 0 & 10 & 1 & (5) & 3 & 17 \\
\hline
\end{tabular}

Table 8.4. Published Chandra and XMM observations of low-luminosity X-ray sources in globular clusters. For each cluster we give the lowest detectable luminosity ( $\mathrm{erg} \mathrm{s}^{-1}$, estimated for the range 0.5-2.5 $\mathrm{keV}$ ), and the estimated numbers of X-ray sources corresponding to luminous low-mass X-ray binaries (BX), low-luminosity low-mass $X$-ray binaries $(F X)$, cataclysmic variables $(C V)$, recycled pulsars (PSX), (for comparison: we list the number of radio pulsars in the column $P S R)$, and magnetically active binaries $(B Y) . \rightarrow 1$ ? $(1 ? \leftarrow)$ indicates that one of the sources in the previous (next) column actually may belong in this column. The final column gives the total number of detected X-ray sources associated with the cluster.

Interestingly, most magnetically active binaries identified with X-ray sources so far have visual magnitudes higher than or equal to the turnoff stars, implying that they are on the main sequence (BY Dra's). Since the maximum X-ray luminosity of a magnetically active binary scales roughly with the surface area of the stars, this implies that the luminosities of the active binaries in globular clusters are low (typically $L_{x}<10^{30} \mathrm{erg} \mathrm{s}^{-1}$ ), compared to systems with giants (RS CVn's), in the Galaxy, which can be up to a hundred times more luminous (Dempsey et al. 1993).

\subsubsection{Comparing clusters}

In comparing the different clusters, the limit to which sources can be detected must be taken into account. Low-luminosity low-mass X-ray binaries with a neutron star tend to be more luminous than cataclysmic variables, which in turn tend to be more luminous than magnetically active binaries. This ordering is reflected in the numbers of currently known cataclysmic variables and magnetically active binaries listed in Table 8.4 as a function of the detection limit.

Another number that is important is the estimated number of close encounters between stars in the globular cluster. Pooley et al. (2003) show that the number of X-ray sources detected in a globular cluster above an observational threshold of $L_{x} \simeq 4 \times 10^{30} \mathrm{erg} \mathrm{s}^{-1}(0.5-6 \mathrm{keV})$ scales quite well with this number, as shown in Figure 8.13. Heinke et al. (2003d) find that the number of cataclysmic variables 
alone (at $L_{x} \gtrsim \times 10^{31} \mathrm{erg} \mathrm{s}^{-1}$ ) possibly increases slower with central density than predicted by proportionality to the number of close encounters.

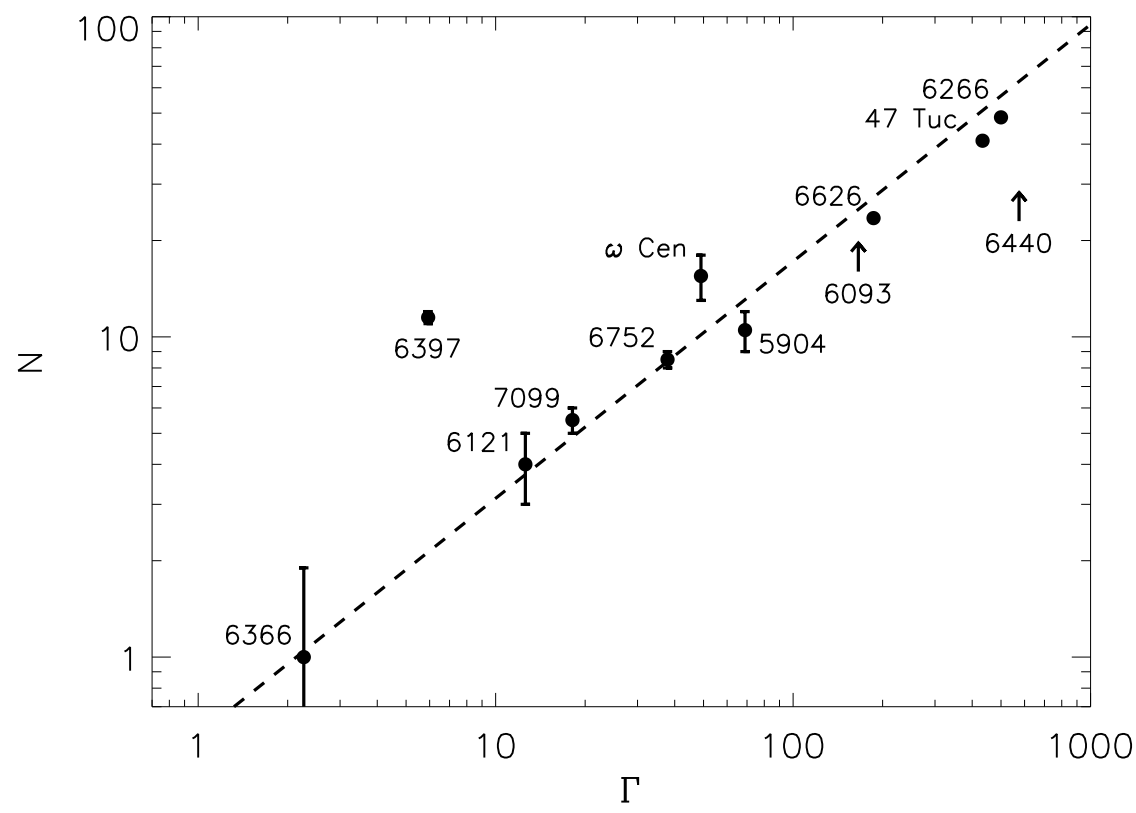

Fig. 8.13. Number $N$ of X-ray sources with $L_{x} \gtrsim 4 \times 10^{30} \operatorname{erg~s}^{-1}(0.5-6 \mathrm{keV})$ detected in globular clusters, as a function of the collision number $\Gamma$. $\Gamma$ is a measure of the number of close encounters between stars in a cluster (see Eqs. 8.5 and 8.6]. The luminosity limit implies that most sources are cataclysmic variables. In general $N$ scales quite well with $\Gamma$, indicating that cataclysmic variables in globular clusters are formed via close encounters between a white dwarf and another star or a binary. Arrows indicate lower limits. NGC 6397 doesn't follow the general trend. From Pooley et al. (2003).

An exception to this scaling is NGC 6397. This cluster has a higher number of neutron star binaries and cataclysmic variables than expected on the basis of its rather low collision number. Remarkably, the number of magnetically active binaries in this cluster is not very high, and this is reflected in a relatively flat X-ray luminosity function (Pooley et al. 2002b). If it is true, as argued by Pooley et al. (2003), that the high number of neutron star binaries and cataclysmic variables in NGC 6397 is due to its being shocked and stripped in multiple passages through the galactic disk and/or near the galactic center, it has to be explained why these mechanisms are more efficient in removing magnetically active binaries than in removing cataclysmic variables and binaries with neutron stars. 


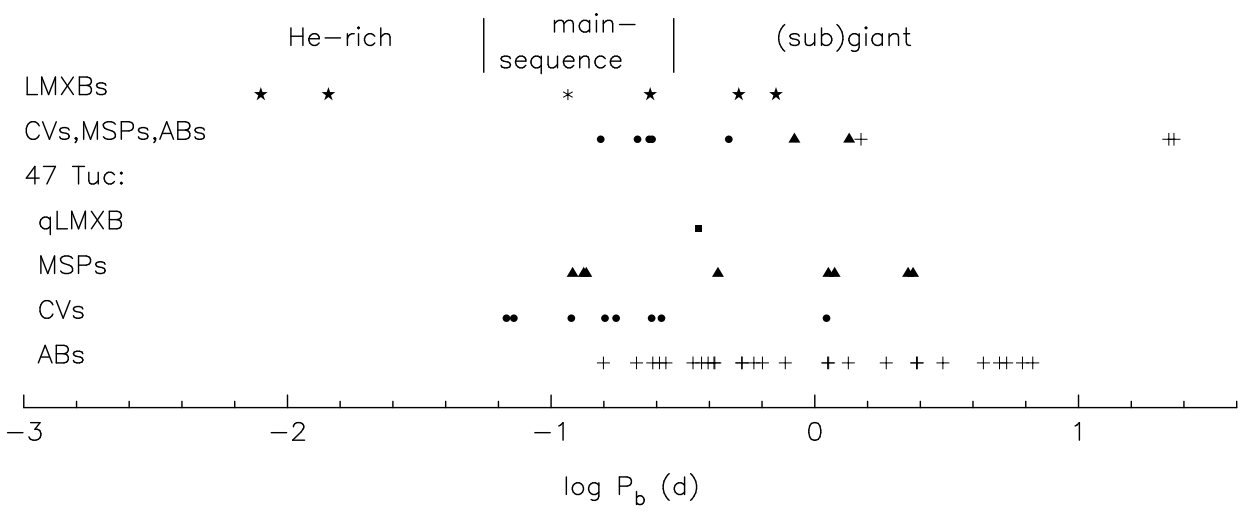

Fig. 8.14. Orbital period distributions of X-ray-detected binaries in globular clusters. Most known orbital periods are for systems in 47 Tuc, and are shown in the lower four rows. The top two rows indicate the luminous X-ray binaries and other binaries in other clusters (with symbols as for 47 Tuc). The period of a cluster source in M31 is shown with a six-pointed star. The period range in which a main-sequence star can fill its Roche lobe is indicated; systems with shorter periods may contain degenerate stars, with longer periods (sub)giants. Periods from Table 8.1; 47 Tuc: Edmonds et al. (2003), Freire et al. (2003), Camilo et al. (2000); other clusters: Bailyn et al. (1996), Deutsch et al. (2000), Neill et al. (2002), Kaluzny 85 Thompson (2002), Kaluzny et al. (1996), D'Amico et al. (2001, 2002); M31: Trudolyubov et al. (2002).

\subsection{Some remarks on evolution and formation}

\subsubsection{Evolution}

A good first indicator of the evolutionary status of a binary is its orbital period (see Chapter 16 by Tauris \& Van den Heuvel and Verbunt 1993 for a more extended discussion of the evolution of X-ray binaries). We show the orbital periods of X-ray emitting binaries in globular clusters in Figure 8.14 Most periods known are for binaries in 47 Tuc. It should be noted that there is a selection effect against the discovery of long-period binaries in optical surveys.

The radius $R$ of the Roche lobe of a star with mass $M$ in a binary with a star of mass $m$, is given in units of the distance $a$ between stars as approximately

$$
\frac{R}{a} \simeq 0.46\left(\frac{M}{M+m}\right)^{1 / 3} \quad \text { for } M<0.8 m
$$

Combining this with the third law of Kepler we find

$$
P_{b} \simeq 8.9 \mathrm{hr}\left(\frac{M_{\odot}}{M}\right)^{1 / 2}\left(\frac{R}{R_{\odot}}\right)^{3 / 2}
$$

i.e. the orbital period gives the average density of a Roche-lobe filling star (cf. §5.3.1).

The radius of a main sequence star is roughly given by $R / R_{\odot} \simeq M / M_{\odot}$ in the mass range of interest here. With main-sequence stars in old globular clusters limited to masses $M \lesssim 0.8 R_{\odot}$, we see that binaries in which mass transfer occurs, i.e. low-mass $\mathrm{X}$-ray binaries and cataclysmic variables, can only have a main sequence star as the 
mass donor provided the orbital period is less than about $7 \mathrm{hr}$. If the orbital period is longer, the donor must be larger than a main-sequence star, i.e. a (sub)giant. It then follows from Figure 8.14 that, with one exception, all cataclysmic variables in globular clusters can have main-sequence donors. The one exception is AKO9, a cataclysmic variable with a slightly evolved donor in 47 Tuc (e.g. Knigge et al. 2003). Of the low-mass X-ray binaries, one may have a main-sequence donor, two binaries must have subgiant donors; the low-luminosity low-mass X-ray binary in 47 Tuc is probably a subgiant close to the main sequence. The orbital periods of most active binaries are long enough that even main-sequence stars near the turnoff mass $\left(0.8 M_{\odot}\right)$ fit well within the Roche lobes; for those with the shorter periods, both stars must have lower masses to be smaller than their Roche lobes. Two of the low-mass X-ray binaries have ultra-short orbital periods; at such short orbital periods the Roche filling star can be a white dwarf. With $R / R_{\odot} \simeq 0.01\left(M / M_{\odot}\right)^{-1 / 3}$, a white dwarf fills its Roche lobe if the orbital period $P_{b} \simeq 48 \mathrm{~s} M_{\odot} / M$.

The evolution of low-mass X-ray binaries and cataclysmic variables with mainsequence donors is driven by the loss of angular momentum $\dot{J}$ from the binary. Writing the angular momentum of the binary as $J_{b}$, one finds that the mass-transfer rate $\dot{M}$ is roughly given by $-\dot{M} / M \sim-\dot{J} / J_{b}$. The loss of angular momentum from gravitational radiation alone is enough to drive mass transfer at a rate of $10^{-10} M_{\odot} y r^{-1}$; higher mass transfer rates, as witnessed by luminosities well in excess of $L_{x} \simeq 10^{36} \mathrm{erg} \mathrm{s}^{-1}$, imply other mechanisms. The loss of angular momentum causes the orbit to shrink, and thus the orbital period to become shorter. In binaries with a (sub)giant donor, the mass transfer rate is very roughly given by the expansion rate of the donor star $-\dot{M} / M \sim \dot{R} / R$. Since the expansion rate of a giant becomes faster as it further ascends the giant branch, this predicts higher mass transfer, i.e. more luminous X-ray emission, for the longest periods. For the two known orbital periods of low-mass X-ray binaries in globular clusters with a subgiant, expansion of the donor predicts a modest mass transfer of $\sim 10^{-10} M_{\odot} y r^{-1}$. The mass transfer, combined with conservation of angular momentum, causes the orbit to expand, and the orbital period to increase. Enhanced loss of angular momentum from a stellar wind has often been invoked to explain large X-ray luminosities, in binaries with main-sequence or subgiant donors, but the actual efficiency of this loss mechanism is not known. It is worth noting that many X-ray sources show large variations in their $\mathrm{X}$-ray luminosity on time scales of decades - the transients are an obvious example - indicating that the current mass transfer rate, even in apparently stable systems, may not be an accurate estimator of mass transfer rate on an evolutionary time scale.

Something is wrong with the simplest description of binary evolution. This follows, e.g., from the orbital period distribution of the recycled radio pulsars. The expansion of a binary with a subgiant donor continues until the core of the giant is denuded of its envelope. By then the orbital period has increased by an order of magnitude. The orbital periods of the radio pulsars in 47 Tuc are less than about $2.5 \mathrm{~d}$, suggesting that little if any expansion has occurred during the mass transfer. On the other hand, some pulsar binaries in globular clusters, such as the pulsar binary in M4, do have periods in excess of hundred days, with fairly circular orbits, showing that expansion is strong in at least some cases.

What about the ultrashort periods? They may have white-dwarf donors; if so, 
their orbital period should be increasing. It has been suggested that a collision between a (sub)giant and a neutron star could lead to expulsion of the giant envelope and leave the neutron star in orbit around the core, which subsequently cools to an under-massive white dwarf. If loss of angular momentum from gravitational radiation pushes the stars closer, mass transfer begins once the white dwarf fills its Roche lobe (Verbunt 1987). Alternatively, it has been suggested that the ultrashort period systems are the outcome of an evolution which starts when a subgiant starts transferring mass to a neutron star in an orbital period less than $\sim 18 \mathrm{hr}$ (Podsiadlowski et al. 2002). Large loss of angular momentum through a stellar wind brings the two stars closer together, and the evolution proceeds to shorter and shorter periods. The minimum period reached through such an evolutionary path is short enough to explain the 11 min period of the LMXB ${ }^{\mathrm{N} S}$ in NGC 6624. It is predicted that this binary has a negative period derivative, as observed. There are two problems with this scenario, however. One is that the loss of angular momentum from the giant, required at the start of the mass transfer to convert orbital expansion into orbital shrinking, is rather high; perhaps implausibly high. Second, none of the evolutions along this scenario computed by Pylyser \& Savonije (1988) reach the shortest periods within a Hubble time, because it already takes very long for a $1 M_{\odot}$ star to fill its Roche lobe in a $16 \mathrm{hr}$ period. Van der Sluys et al. (2005) investigate this in more detail and find that only binaries in narrow ranges of initial orbital periods and component masses evolve to ultrashort periods within a Hubble time, and that these binaries only spend a small fraction of their life at ultrashort periods; they conclude that no significant population of ultrashort-period binaries in globular clusters can be produced through this evolution channel. The most likely mechanism to produce bright X-ray sources with ultrashort orbital periods is mass transfer from an intermediate-mass donor leading to a common envelope, some time in the past history of the globular cluster (Davies \& Hansen 1998, Rasio et al. 2000). The result is a binary of the neutron star and the core of the giant, which cools into a white dwarf. In the course of several billion years, gravitational radiation may bring the system into contact.

\subsubsection{Some specific systems}

The orbital period for the low-luminosity low-mass X-ray binary 47 Tuc X5 is too long for a Roche-lobe filling main sequence donor star with a mass less than the turnoff mass of $0.8 M_{\odot}$. Edmonds et al. (2002b) therefore conclude that the star is smaller than its Roche lobe. We suggest an alternative possibility that the system hosts a $0.8 M_{\odot}$ subgiant donor that has recently started to transfer matter to a $1.4 M_{\odot}$ neutron star. The donor has not yet transferred much of its envelope mass: a low donor mass in an $8.666 \mathrm{hr}$ orbit implies a Roche lobe for the donor that is too small to hold a subgiant. The system is very sub-luminous for a subgiant: this is expected for a donor that is losing mass.

PSR 47 Tuc W (Chandra source 29) is a pulsar accompanied by an object whose location in the color-magnitude diagram indicates that it is too big for a white dwarf and too small for a main-sequence star. The orbital lightcurve shows clear heating by the pulsar (Edmonds et al. 2002a). If a main-sequence star is heated at constant radius, it moves up and to the left in a color-magnitude diagram, to a location below the main-sequence. If the companion to PSR 47 Tuc W is of this nature, its position 
about 5 magnitudes below turnoff indicates a very low mass, of an $\mathrm{M}$ dwarf. This poses an interesting puzzle for the evolutionary history: if the $\mathrm{M}$ dwarf was in the binary from the start, it was too small to transfer mass to the neutron star and spin it up. If on the other hand the main-sequence star was captured by the pulsar tidally or via an exchange encounter, the orbit should be eccentric initially; the question is whether tidal dissipation can circularize the orbit and heat the $\mathrm{M}$ dwarf to its current position.

PSR NGC $6397 \mathrm{~A}$ is another pulsar accompanied by a low-mass $\left(\sim 0.25 M_{\odot}\right)$ companion (Ferraro et al. 2003). In this case the companion lies somewhat to the right of the turnoff, at a radius of $1.6(2) R_{\odot}$ and luminosity $2.0(4) L_{\odot}$; notwithstanding the proximity of an energetic radio pulsar, the companion shows no sign of heating (Orosz \& van Kerkwijk 2003). The position of the companion in the color-magnitude diagram is hard to explain. Orosz \& van Kerkwijk invoke a stellar collision, causing a slightly evolved star near the turnoff to lose most of its envelope.

\subsubsection{Black holes}

The absence of known very luminous $\left(L_{x} \geq 10^{38.5} \mathrm{erg} \mathrm{s}^{-1}\right.$,say) low-mass X-ray binaries with a black hole in globular clusters of our Galaxy has led to the suggestion that black holes are efficiently ejected from globular clusters through dynamical processes (Kulkarni et al. 1993; Portegies Zwart \& McMillan 2000). The discovery of very luminous, soft X-ray sources in globular clusters in other galaxies shows that X-ray binaries with black holes probably exist in globular clusters (see $\S 8.3)$.

There is no evidence that M15 contains an intermediate mass black hole; an upper limit for the mass of about $10^{3} M_{\odot}$ can be set both from an analysis of pulsar accelerations in this cluster, and from an analysis of radial velocities of stars close to the center (Phinney 1992; Gerssen et al. 2003). A case has been made for a binary in NGC 6752 of two black holes, of which at least one has an intermediate mass (Colpi et al. 2002). The argument for this is the presence of a white-dwarf/radiopulsar binary in the outskirts of the cluster, which most likely was ejected from the cluster core. If the binary was ejected with the white dwarf companion to the pulsar already formed, the very small eccentricity of its orbit implies that the orbit of the other binary involved in the scattering was much larger. To still produce an ejection velocity for the pulsar binary high enough for it to reach the outer cluster region then requires at least one black hole with a mass $\sim 100 M_{\odot}$ in the scattering binary (Colpi et al. 2002). To solidify the case for a binary black hole it would have to be demonstrated that the pulsar indeed belongs to NGC 6752 (as is probable), and that the pulsar binary was ejected before the formation of the white dwarf (which is not obvious). The optical identification of the white dwarf companion to this pulsar shows that the white dwarf is young compared to the age of the globular cluster; this strengthens the case for a scenario in which a binary consisting of a main-sequence star and a neutron star was ejected from the cluster core, and subsequent evolution of the main-sequence star led to circularization of the orbit (Bassa et al. 2003). 


\subsubsection{Formation}

The rate at which stars with number density $n$ encounter target stars with number density $n_{c}$ in a cluster with dispersion velocity $v$ is given by (e.g. Hut \& Verbunt 1983):

$$
\Gamma \propto \int n_{c} n A v d V \propto \int \frac{n_{c} n R}{v} d V \propto \frac{\rho_{o}^{2} r_{c}^{3}}{v} R
$$

where $A$ is the interaction cross section (proportional to $R / v^{2}$ because of gravitational focusing), $R$ the radius of the star, $\rho_{o}$ is the central mass density of the cluster and $r_{c}$ its core radius. Because the number densities of stars drop rapidly with distance from the cluster center, the integral over volume $d V$ can be approximated by multiplying the central encounter rate with the volume of the cluster core. An analogous equation gives the exchange encounter rate

$$
\Gamma_{e} \propto \int n_{c} n_{b} A_{b} v d V \propto \int \frac{n_{c} n_{b} a}{v} d V \propto \frac{\rho_{o}^{2} r_{c}^{3}}{v} a
$$

where $n_{b}$ is the number of binaries per unit volume, and $a$ the semi-major axis of the binary. The ratio of tidal capture to exchange encounters is roughly

$$
\frac{\Gamma}{\Gamma_{e}} \sim \frac{R}{a} \frac{n}{n_{b}}
$$

The velocity dispersion $v$ is related to the core mass and radius through (a specific version of) the virial theorem (King 1966):

$$
v \propto \sqrt{\rho_{o}} r_{c}
$$

Therefore (Verbunt 2003)

$$
\Gamma \propto \rho_{o}{ }^{1.5} r_{c}{ }^{2} R \quad \text { and } \quad \Gamma_{e} \propto \rho_{o}{ }^{1.5} r_{c}{ }^{2} a
$$

Because neutron stars are formed with appreciable velocities, a cluster with a high mass is expected to retain a higher fraction of the neutron stars that are formed in it than a cluster with a low mass. In a cluster with strong mass segregation, virtually all the neutron stars will have migrated to the core. Thus a massive cluster with strong mass segregation is expected to have a much higher central number density of neutron stars than a low-mass little-segregated cluster. Thus, the ratio $n_{c} / \rho_{o}$ for neutron stars, and through this the proportionality constant for the last members of Eqs. 8.5 and 8.6 will vary widely between clusters (Verbunt \& Meylan 1988). On the other hand, white dwarfs are always retained upon formation, and due to their lower masses are less affected by mass segregation. This is probably the reason that the relation between the number of X-ray sources (mainly cataclysmic variables) and $\Gamma$ is as narrow as shown in Figure 8.13 .

Due to the large number density of stars in a cluster core, an appreciable fraction of neutron stars in that core may be involved in a close encounter with a single star or with a binary. The formation of tidal bulges during passage of a neutron star within $\sim 3$ times the radius of a main-sequence star drains enough energy from the relative motion of the two stars to bind them in a binary. This process is called tidal capture (Fabian et al. 1975). Whether it is efficient in the formation of a binary with 
a neutron star is under debate, because of the large amount of energy residing in the initially very eccentric orbit of the newly formed binary. If the orbit circularizes rapidly because of tides on the main sequence star, the energy released is enough to (almost) destroy the main sequence star (Ray et al. 1987, Verbunt 1994). Rapid circularization can be avoided if the energy exchange between tides and orbit is chaotic, as is likely in a highly eccentric orbit (Mardling 1995). Mass loss from the main-sequence star due to tidal heating may further limit the damage to the deeper layers of the star.

A neutron star can also be exchanged into a pre-existing binary when it takes the place of one of the binary members in an exchange encounter (Hills 1976). Which of the two mechanisms is more important depends on the number of binaries present in the core and on their orbital period distribution; as well as on the efficiency of the tidal capture process.

If a binary is of a type that very rarely results from the evolution of a primordial binary, then its presence in a globular cluster may be ascribed to formation via a close encounter. Such is the case for binaries with a neutron star. If a binary is very frequently formed from a primordial binary, then it is likely to be primordial also when present in a globular cluster. This is the case for magnetically active close binaries. Cataclysmic variables are somewhere in between, and thus in clusters can be formed both via close encounters and via evolution of a primordial binary. Figure 8.13 shows that the number of binaries with $L_{x} \gtrsim 4 \times 10^{30} \mathrm{erg} \mathrm{s}^{-1}$ scales well with the number of encounters in a cluster. Since most binaries with such luminosities are cataclysmic variables, this implies that most cataclysmic variables are in fact formed via close encounters. One reason for this is that evolution from a primordial binary into a cataclysmic variable passes through a stage in which the binary is very wide; such a wide binary is easily unbound in a globular cluster by a passing star and the formation of a cataclysmic variable is prevented (Davies 1997). If the number of cataclysmic variables increases more slowly with central density than as $\rho_{o}^{1.5}$, as suggested by Heinke et al. (2003d), this could suggest that primordial binaries do still contribute to the formation of cataclysmic variables. Remarkably, Jordán et al. (2004) find that the probability for a globular cluster associated with NGC 4486 (M 87) to harbor a bright X-ray source also scales with a lower power of $\rho_{o}$ than the collision number, i.e. roughly as $\propto \Gamma \rho_{o}^{-0.5}$.

Looking now at the period distribution of the cataclysmic variables and low-mass $\mathrm{X}$-ray binaries in globular clusters, we see that their periods are short, $\lesssim 1 \mathrm{~d}$. This may indicate that they are formed at short periods, which hints at tidal capture as the main formation process. Some care is necessary before one jumps to conclusions, however. Mass transfer in wide binaries tends to be faster, and thus wide binaries live shorter, and will be less numerous even if their formation rate is the same as that of short binaries. Also, longer periods are more difficult to measure, and some of the many binaries with unknown periods may have long periods. In addition, a wide binary with a neutron star or white dwarf can become closer via encounters with field stars. We doubt that this process is sufficiently efficient, given the observed presence in 47 Tuc of active binaries with periods up to ten days that apparently have avoided further shrinking of their orbits. On the whole we tend to conclude that rumors of 
the death of the tidal capture model for the formation of binaries with a neutron star and of cataclysmic variables have been much exaggerated.

That exchange encounters do occur in globular clusters is evident from the wide pulsar binaries, such as M 4 PSR A $\left(P_{b}=191 \mathrm{~d}\right.$, Thorsett et al. 1999) and M 53 PSR A (255 d, Kulkarni et al. 1991). These are found in clusters with a relatively low central density, which allows long period binaries to survive (e.g. Verbunt 2003). They must have evolved from binaries with initial periods too long to be formed by tidal capture, in which the neutron star can thus only have entered via an exchange encounter. (M 15 PSR C is an eccentric binary of two neutron stars in the outskirts of M 15, and is another product of an exchange encounter: Phinney \& Sigurdsson 1991.)

Acknowledgements We are very grateful for comments, suggestions and help from Lorella Angelini, Keith Ashman, Pauline Barmby, Cees Bassa, Boris Dirsch, Rosanne Di Stefano, Josh Grindlay, Bill Harris, Piet Hut, Andres Jordán, Arunav Kundu, Erik Kuulkers, Tom Maccarone, Dave Pooley, Katherine Rhode, Craig Sarazin, Rudy Wijnands, and Steve Zepf.

\section{References}

1. Anderson, J., Cool, A., King, I. 2003, ApJ, 597, L137

2. Anderson, S., Margon, B., Deutsch, E., Downes, R., \& Allen, R. 1997, ApJ, 482, L69

3. Angelini, L., Loewenstein, M., \& Mushotzky, R. 2001, ApJ, 557, L35

4. Ashman, K. \& Zepf, S. 1992, ApJ, 384, 50

5. - 1998, Globular cluster systems (Cambridge: Cambridge U.P.)

6. Aurière, M., Le Fèvre, O., \& Terzan, A. 1984, A\&A, 138, 415

7. Bahcall, J. \& Wolf, R. 1976, ApJ, 209, 214

8. Bailyn, C., Grindlay, J., \& Garcia, M. 1990, ApJ, 357, L35

9. Bailyn, C., Rubenstein, E., Slavin, S., \& et al. 1996, ApJ, 473, L31

10. Barmby, P. 2003, in Extragalactic Globular Cluster Systems, ESO Workshop, 143

11. Barmby, P. \& Huchra, J. 2001, AJ, 122, 2458

12. Bassa, C. \& Stappers, B. 2004, A\&A, 425, 1143

13. Bassa, C., Verbunt, F., Van Kerkwijk, M., \& Homer, L. 2003, A\&A, 409, L31

14. Bassa, C., Pooley, D. \& Homer, L. \& et al. 2004, ApJ, 609, 755

15. Becker, W., Swartz, D., Pavlov, G., \& et al. 2003, ApJ, 594, 798

16. Bellazzini, M., Pasquali, A., Federici, L., \& et al. 1995, ApJ, 439, 687

17. Bildsten, L. \& Rutledge, R. 2000, ApJ, 541, 908

18. Bildsten, L. \& Deloye, C. 2004, ApJ, 607, L119

19. Blanton, E., Sarazin, C., \& Irwin, J. 2001, ApJ, 552, 106

20. Bridges, T. \& Hanes, D. 1990, AJ, 99, 1100

21. Callanan, P., Drake, J., \& Fruscione, A. 1999, ApJ, 521, L125

22. Camilo, F., Lorimer, D., Freire, P., Lyne, A., \& Manchester, R. 2000, ApJ, 535, 975

23. Canizares, C. \& Neighbours, J. 1975, ApJ, 199, L97

24. Carson, J., Cool, A., \& Grindlay, J. 2000, ApJ, 532, 461

25. Chou, Y. \& Grindlay, J. 2001, ApJ, 563, 934

26. Clark, G. 1975, ApJ, 199, L143

27. Clark, G., Markert, T., \& Li, F. 1975, ApJ, 199, L93

28. Cohen, J., Blakeslee, J., \& Côté, P. 2003, ApJ, 592, 866

29. Colpi, M., Possenti, A., \& Gualandris, A. 2002, ApJ, 570, L85

30. Cool, A., Grindlay, J., Krockenberger, M., \& Bailyn, C. 1993, ApJ, 410, L103 
31. Cool, A., Grindlay, J., Cohn, H., Lugger, P., \& Slavin, S. 1995, ApJ, 439, 695

32. Cool, A., Grindlay, J., Cohn, H., Lugger, P., \& Bailyn, C. 1998, ApJ, 508, L75

33. Cool, A., Haggard, D., \& Carlin, J. 2002, in $\omega$ Cen, a unique window into astrophysics, ed.

F. van Leeuwen, J. Hughes, \& G. Piotto (ASP Conf. Ser. 265), 277-288

34. Côté, P. 1999, AJ, 118, 406

35. Côté, P., West, M., \& Marzke, R. 1999, ApJ, 567, 853

36. D'Amico, N., Possenti, A., Manchester, D. \& et al. 2001, ApJ, 561, L89

37. D'Amico, N., Possenti, A., Fici, L., \& et al. 2002, ApJ, 570, L89

38. Davies, M. 1997, MNRAS, 288, 117

39. Davies, M. \& Hansen, B. 1998, MNRAS, 301, 15

40. Dempsey, R., Linsky, J., Fleming, T., \& Schmitt, J. 1993, ApJS, 86, 599

41. Deutsch, E., Anderson, S., Margon, B., \& Downes, R. 1996, ApJ, 472, L97

42. 一. 1998, ApJ, 493, 765

43. Deutsch, E., Margon, B., \& Anderson, S. 2000, ApJ, 530, L21

44. di Stefano, R., Kong, A., Garcia, M., \& et al. 2002, ApJ, 570, 618

45. di Stefano, R., Kong, A., VanDalfsen, M., \& et al. 2003, ApJ, 599, 1067

46. Dirsch, B., Richtler, T., Geisler, D., \& et al. 2003, AJ, 125, 1908

47. Dotani, T., Inoue, H., Murakami, T., \& et al. 1990, Nature, 347, 534

48. Dotani, T., Asai, K., \& Greiner, J. 1999, Publ. Astr. Soc. Japan, 51, 519

49. Edmonds, P., Grindlay, J., Cool, A., Cohn, H., Lugger, P., \& Bailyn, C. 1999, ApJ, 516, 250

50. Edmonds, P., Gilliland, R., Camilo, F., Heinke, C., \& Grindlay, J. 2002a, ApJ, 579, 741

51. Edmonds, P., Heinke, C., Grindlay, J., \& Gilliland, R. 2002b, ApJ, 564, L17

52. Edmonds, P., Gilliland, R., Heinke, C., \& Grindlay, J. 2003, ApJ, 596, 1177 \& 1197

53. Edmonds, P., Kahabka, P. \& Heinke, C. 2004, ApJ, 611, 413

54. Fabian, A., Pringle, J., \& Rees, M. 1975, MNRAS, 172, 15p

55. Ferraro, F., Paltinieri, B., Fusi Pecci, F., Rood, R., \& Dorman, B. 1997, MNRAS, 292, L45

56. Ferraro, F., Paltinieri, B., Rood, R., Fusi Pecci, F., \& Buonanno, R. 2000, ApJ, 537, 312

57. Ferraro, F., Possenti, A., D'Amico, N., \& Sabbi, E. 2001, ApJ, 561, L93

58. Ferraro, F., Sabbi, E., Gratton, R., \& et al. 2003, ApJ, 584, L13

59. Forbes, D., Grillmair, C., \& Smith, R. 1997, AJ, 113, 1648

60. Forman, W. \& Jones, C. 1976, ApJ, 207, L177

61. Forman, W., Jones, C., \& Tananbaum, H. 1976, ApJ, 207, L25

62. Fox, D., Lewin, W., Margon, B., van Paradijs, J., \& Verbunt, F. 1996, MNRAS, 282, 1027

63. Freire, P., Camilo, F., Kramer, M., \& et al. 2003, MNRAS, 340, 1359

64. Geffert, M. 1998, A\&A, 340, 305

65. Gendre, B., Barret, D., \& Webb, N. 2003a, A\&A, 400, 521

66. - 2003b, A\&A, 403, L11

67. Gerssen, J., van der Marel, R., Gebhardt, K., \& et al. 2003, AJ, 125, 376

68. Giacconi, R., Murray, S., Gursky, H., \& et al. 1972, ApJ, 178, 281

69. Giacconi, R., Murray, S., Gursky, H., \& et al. 1974, ApJS, 27, 37

70. Gómez, M., Richtler, T., Infante, L., \& Drenkhahn, G. 2001, A\&A, 371, 875

71. Goodman, J. \& Hut, P. 1989, Nature, 339, 40

72. Goudfrooij, P., Alonso, M., Maraston, C., \& Minniti, D. 2001, MNRAS, 328, 237

73. Grimm, H., Gilfanov,M., Sunyaev, R. 2002, A\&A, 391, 923

74. Grindlay, J. 1987, in The Origin and Evolution of Neutron Stars, IAU Symposium No. 125, ed.

D. Helfand \& J.-H. Huang (Dordrecht: Reidel), 173-185

75. Grindlay, J. 1988, in Globular cluster systems in galaxies, IAU Symposium No. 126, ed. J.

Grindlay \& G. Davis Philip (Dordrecht: Reidel), 347-366

76. Grindlay, J. 1992, in X-ray binaries and recycled pulsars, NATO ASI C. 377, eds. E. van den

Heuvel \& S. Rappaport (Dordrecht: Kluwer), 365

77. Grindlay, J. \& Hertz, P. 1985, in Cataclysmic Variables and Low Mass X-ray Binaries, ed.

D. Lamb \& J. Patterson (Dordrecht: Reidel), 79-91

78. Grindlay, J., Gursky, H., Schnopper, H., \& et al. 1976, ApJ, 205, L127

79. Grindlay, J., Hertz, P., Steiner, J., Murray, S., \& Lightman, A. 1984, ApJ, 282, L13

80. Grindlay, J., Cool, A., Callanan, P., Bailyn, C., Cohn, H., \& Lugger, P. 1995, ApJ, 455, L47

81. Grindlay, J., Heinke, C., Edmonds, P., , \& Murray, S. 2001a, Science, 292, 2290

82. Grindlay, J., Heinke, C., Edmonds, P., , Murray, S., \& Cool, A. 2001b, ApJ, 563, L53 
83. Grindlay, J., Camilo, F., Heinke, C., Edmonds, P., Cohn, H., \& Lugger, P. 2002, ApJ, 581, 470 84. Guainazzi, M., Parmar, A., \& Oosterbroek, T. 1999, A\&A, 349, 819

85. Gunn, J. \& Griffin, R. 1979, AJ, 84, 752

86. Gursky, H. July 1973, Lecture presented at the NASA Advanced Study Institute on Physics of

Compact Objects, Cambridge, UK

87. Haggard, D., Cool, A., Anderson, J., Edmonds, P. et al. 2004, ApJ, 613, 512

88. Hakala, P., Charles, P., Johnston, H., \& Verbunt, F. 1997, MNRAS, 285, 693

89. Harris, W. 1991, ARA\&A, 29, 543

90. - . 1996, AJ, 112, 1487

91. Harris, W. \& van den Bergh, S. 1981, AJ, 86, 1627

92. Heinke, C., Edmonds, P., \& Grindlay, J. 2001, ApJ, 562, 363

93. Heinke, C., Edmonds, P., Grindlay, J., Lloyd, D., Cohn, H., \& Lugger, P. 2003a, ApJ, 590, 809

94. Heinke, C., Grindlay, J., Lloyd, D., \& Edmonds, P. 2003b, ApJ, 588, 452

95. Heinke, C., \& Grindlay, J., Edmonds, P. \& et al. 2003c, ApJ, 598, 516

96. Heinke, C., \& Grindlay, J., Lugger, P.. \& et al. 2003d, ApJ, 598, 501

97. Hénon, M., 1961, Ann. d'Astroph., 24, 369

98. Hertz, P. \& Grindlay, J. 1983, ApJ, 275, 105

99. Hertz, P. \& Wood, K. 1985, ApJ, 290, 171

100. Hertz, P., Grindlay, J., \& Bailyn, C. 1993, ApJ, 410, L87

101. Hills, J. 1976, MNRAS, 175, 1p

102. Hoffman, J., Lewin, W., \& Doty, J. 1977a, MNRAS, 179, 57P

103. —. 1977b, ApJ, 217, L23

104. Hoffman, J., Marshall, H., \& Lewin, W. 1978, Nature, 271, 630

105. Hoffman, J., Cominsky, L., \& Lewin, W. 1980, ApJ, 240, L27

106. Homer, L., Charles, P., Naylor, T., \& et al. 1996, MNRAS, 282, L37

107. Homer, L., Anderson, S., Margon, B., Deutsch, E., \& Downes, R. 2001a, ApJ, 550, L155

108. Homer, L., Deutsch, E., Anderson, S., \& Margon, B. 2001b, AJ, 122, 2627

109. Homer, L., Anderson, S., Margon, B., Downes, R., \& Deutsch, E. 2002, AJ, 123, 3255

110. Hut, P. \& Verbunt, F. 1983, Nature, 301, 587

111. Hut, P., McMillan, S., Goodman, J., \& et al. 1992, PASP, 104, 981

112. Iben, I., Tutukov, A., \& Fedorova, A. 1997, ApJ, 486, 955

113. Ilovaisky, S., Aurière, M., Koch-Miramond, L., \& et al. 1993, A\&A, 270, 139

114. in 't Zand, J., Verbunt, F., Heise, J., \& et al. 1998, A\&A, 329, L37

115. in 't Zand, J., Verbunt, F., Strohmayer, T., \& et al. 1999, A\&A, 345, 100

116. in 't Zand, J., Bazzano, A., Cocchi, M., \& et al. 2000, A\&A, 355, 145

117. in 't Zand, J., van Kerkwijk, M., Pooley, D., \& et al. 2001, ApJ, 563, L41

118. in 't Zand, J., Hulleman, . F., Markwardt, C., \& et al. 2003, A\&A, 406, 233

119. Ioannou, Z., van Zyl, L., Naylor, T., \& et al. 2003, A\&A, 399, 211

120. Irwin, J. \& Bregman, J. 1999, ApJ, 510, L21

121. Jeltema, T., Sarazin, C., Buote, D., \& Garmire, G. 2003, ApJ, 585, 756

122. Jernigan, J. \& Clark, G. 1979, ApJ, 231, L125

123. Johnston, H., Verbunt, F., \& Hasinger, G. 1994, A\&A, 289, 763

124. - 1995, A\&A, 298, L21

125. - 1996, A\&A, 309, 116

126. Jordán, A., Côté, P., Ferrarese, L., et al. 2004 ApJ, 613, 279

127. Kaluzny, J. \& Thompson, I. 2002, AJ, 125, 2534

128. Kaluzny, J., and Kubiak, M., Szymanski, M., et al. 1996, A\&AS, 120, 139

129. Katz, J. 1975, Nature, 253, 698

130. Kim, D.-W. \& Fabbiano, G. 2003, ApJ, 586, 826

131. Kim, D.-W. \& Fabbiano, G. 2004, ApJ, 611, 846

132. King, I. 1966, AJ, 71, 64

133. King, I., Stanford, S., Albrecht, R., \& et al. 1993, ApJ, 413, L117

134. Kissler-Patig, M. 1997, A\&A, 319, 83

135. Knigge, C., Zurek, D., Shara, M., Long, K., \& Gilliland, R. 2003, ApJ, 599, 1320

136. Kraft, R., Forman, W., Jones, C., \& et al. 2000, ApJ, 531, L9

137. Kraft, R., Kregenov, J., Forman, W., Jones, C., \& Murray, S. 2001, ApJ, 560, 675

138. Kulkarni, S., Goss, W., Wolszczan, A., \& Middleditch, J. 1990, ApJ, 363, L5 
139. Kulkarni, S., Anderson, S., Prince, T., \& Wolszczan, A. 1991, Nature, 349, 47

140. Kulkarni, S., Hut, P., \& McMillan, S. 1993, Nature, 364, 421

141. Kundu, A. \& Whitmore, B. 2001a, AJ, 121, 2950

142. —. 2001b, AJ, 122, 1251

143. Kundu, A., Maccarone, T., \& Zepf, S. 2002, ApJ, 574, L5

144. Kundu, A., Maccarone, T., Zepf, S., \& Puzia, T. 2003, ApJ, 589, L81

145. Kuulkers, E., den Hartog, P., in 't Zand, J., \& et al. 2003, A\&A, 399, 663

146. Larsen, S., Brodie, J., Beasley, M., \& et al. 2003, ApJ, 585, 767

147. Lewin, W. 1980, in Globular Clusters, ed. D. Hanes \& B. Madore (Cambridge: Cambridge U.P.), 315

148. Lewin, W. \& Joss, P. 1983, in Accretion-driven stellar X-ray sources, ed. W. Lewin \&

E. van den Heuvel (Cambridge: Cambridge U.P.), 41-115

149. Lewin, W., Doty, J., Clark, G., and et al. 1976, ApJ, 207, L95

150. Lewin, W., van Paradijs, J., \& Taam, R. 1993, Space Sci. Rev., 62, 223

151. Lewin, W., van Paradijs, J., \& Taam, R. 1995, in X-ray binaries, ed. W. Lewin, J. van

Paradijs, \& E. van den Heuvel (Cambridge: Cambridge U.P.), 175-232

152. Lyne, A., Brinklow, A., Middleditch, J., \& et al. 1987, Nature, 328, 399

153. Maccarone, T., Kundu, A., \& Zepf, S. 2003, ApJ, 586, 814

154. 一. 2004, ApJ, 606, 430

155. Magnier, E., Lewin, W., van Paradijs, J., \& et al. 1992, A\&AS, 96, 379

156. Makishima, K., Ohashi, T., Inoue, H., et al. 1981, ApJ, 247, L23

157. Mardling, R. 1995, ApJ, 450, 722,732

158. Markert, T., Backman, D., Canizares, C., Clark, G., \& Levine, A. 1975, Nature, 257, 32

159. Masetti, N. 2002, A\&A, 381, L45

160. McLaughlin, D., Harris, W., \& Hanes, D. 1994, ApJ, 422, 486

161. Minniti, D., Rejkuba, M., Funes, J., Akiyama, S. 2004, ApJ 600, 716

162. Mirabel, I. \& Rodrigues, I. 2003, A\&A, 398, L25

163. Mirabel, I., Dhawan, V., Mignami, R., \& et al. 2001, Nature, 413, 139

164. Moore, C., Rutledge, R., Fox, D., \& et al. 2000, ApJ, 532, 1181

165. Naylor, T., Charles, P., Hassall, B., Raymond, J., \& Nassiopoulos, G. 1992, MNRAS, 255, 1

166. Neill, J., Shara, M., Caulet, A., \& Buckley, D. 2002, AJ, 123, 3298

167. Oosterhoff, P. T. 1941, Ann. Sternwarte Leiden, 17, 1

168. Orosz, J. \& van Kerkwijk, M. 2003, A\&A, 397, 237

169. Paresce, F. \& de Marchi, G. 1994, ApJ, 427, L33

170. Paresce, F., de Marchi, G., \& Ferraro, F. 1992, Nature, 360, 46

171. Parmar, A., Stella, L., \& Giommi, P. 1989, A\&A, 222, 96

172. Parmar, A., Oosterbroek, T., Sidoli, L., Stella, L., \& Frontera, F. 2001, A\&A, 380, 490

173. Perrett, K., Hanes, D., Butterworth, S., \& et al. 1997, AJ, 113, 895

174. Phinney, E. 1992, Phil. Trans. R. Soc. London A, 341, 39

175. Phinney, E. \& Sigurdsson, S. 1991, Nature, 349, 220

176. Piotto, G. \& Zoccali, M. 1999, A\&A, 345, 485

177. Piro, A. \& Bildsten, L. 2002, ApJ, 571, L103

178. Podsiadlowski, P., Rappaport, S., \& Pfahl, E. 2002, ApJ, 565, 1107

179. Pooley, D., Lewin, W., Homer, L., Verbunt, F., \& et al. 2002a, ApJ, 569, 405

180. Pooley, D., Lewin, W., Verbunt, F., Homer, L., \& et al. 2002b, ApJ, 573, 184

181. Pooley, D., Lewin, W., Anderson, S., \& et al. 2003, ApJ, 591, L131

182. Portegies Zwart, S. \& McMillan, S. 2000, ApJ, 528, 17

183. Predehl, P., Hasinger, G., \& Verbunt, F. 1991, A\&A, 246, L21

184. Puzia, T., Saglia, R., Kissler-Patig, M., \& et al. 2002, A\&A, 391, 453

185. Pylyser, E. \& Savonije, G. 1988, A\&A, 191, 57

186. Rajagopal, M. \& Romani, R. 1996, ApJ, 461, 327

187. Randall, S., Sarazin, C., \& Irwin, J. 2003, ApJ, 600, 729

188. Rappaport, S., Dewey, D., Levine, A., \& Macri, L. 1994, ApJ, 423, 633

189. Rasio, F., Pfahl, E., \& Rappaport, S. 2000, ApJ, 532, L47

190. Ray, A., Kembhavi, A., \& Antia, H. 1987, A\&A, 184, 164

191. Rhode, K. \& Zepf, S. 2001, AJ, 121, 210

192. —. 2004, AJ, 127, 302 
193. Rutledge, R., Bildsten, L., Brown, E., Pavlov, G., \& Zavlin, V. 1999, ApJ, 514, 945

194. - 2002, ApJ, 578, 405

195. Saito, Y., Kawai, N., Kamae, T., \& et al. 1997, ApJ, 477, L37

196. Sansom, A., Dotani, T., Asai, K., \& Lehto, H. 1993, MNRAS, 262, 429

197. Sarazin, C., Irwin, J., \& Bregman, J. 2000, ApJ, 544, L101

198. - . 2001, ApJ, 556, 533

199. Sarazin, C., Irwin, J., Rood, R., \& et al. 1999, ApJ, 524, 220

200. Sarazin, C., Kundu, A., Irwin, J., \& et al. 2003, ApJ, 595, 743

201. Seward, F., Page, C., Turner, M., \& Pounds, K. 1976, MNRAS, 175, 39P

202. Shara, M., Bergeron, L., Gilliland, R., Saha, A., \& Petro, L. 1996, ApJ, 471, 804

203. Sidoli, L., Parmar, A., Oosterbroek, T., \& et al. 2001, A\&A, 368, 451

204. Sivakoff, G., Sarazin, C., \& Irwin, J. 2003, ApJ, 599, 218

205. Skinner, G., Willmore, A., Eyles, C., \& et al. 1987, Nature, 330, 544

206. Stella, L., Priedhorsky, W., \& White, N. 1987, ApJ, 312, L17

207. Supper, R., Hasinger, G., Pietsch, W., \& et al. 1997, ApJ, 317, 328

208. Sutantyo, W. 1975, A\&A, 44, 227

209. Swank, J., Becker, R., Boldt, E., \& et al. 1977, ApJ, 212, L73

210. Sztajno, M., Fujimoto, M., van Paradijs, J., \& et al 1987, MNRAS, 226, 39

211. Thorsett, S., Arzoumanian, Z., Camilo, F., \& Lyne, A. 1999, ApJ, 523, 763

212. Tomsick, J., Corbel, S., Fender, R., et al. 2003, ApJ, 597, L133

213. Trudolyubov, S., Borozdin, K., Priedhorsky, W., \& et al. 2002, ApJ, 581, L27

214. van den Heuvel, E. 1983, in Accretion-driven stellar X-ray sources, ed. W. Lewin \&

E. van den Heuvel (Cambridge: Cambridge U.P.), 303-341

215. van der Klis, M., Hasinger, G., Verbunt, F. et al. 1993, A\&A, 279, L21

216. van der Sluys, M., Verbunt, F., \& Pols, O., 2005, A\&A, 431, 647

217. van Paradijs, J. 1978, Nature, 274, 650

218. van Paradijs, J. \& McClintock, J. 1994, A\&A, 290, 133

219. van Paradijs, J., Verbunt, F., Shafer, R., \& Arnaud, K. 1987, A\&A, 182, 47

220. van Speybroeck, L., Epstein, A., Forman, W., et al. 1979, ApJ, 234, L45

221. Verbunt, F. 1987, ApJ, 312, L23

222. - . 1993, ARA\&A, 31, 93

223. - . 1994, A\&A, 285, L21

224. - . 1996, in Compact stars in binaries, IAU Symp. 165, ed. J. van Paradijs, E. van den

Heuvel, \& E. Kuulkers (Dordrecht: Kluwer Academic Publishers), 333-339

225. - . 2001, A\&A, 368, 137

226. - . 2003, in New horizons in globular cluster astronomy, ed. G. Piotto, G. Meylan,

G. Gjorgovski, \& M. Riello (ASP Conf. Ser. 296), 245-254

227. Verbunt, F. \& Hasinger, G. 1998, A\&A, 336, 895

228. Verbunt, F. \& Hut, P. 1987, in The Origin and Evolution of Neutron Stars, IAU Symposium

No. 125, ed. D. Helfand \& J.-H. Huang (Dordrecht: Reidel), 187-197

229. Verbunt, F. \& Johnston, H. 2000, A\&A, 358, 910

230. Verbunt, F. \& Meylan, G. 1988, A\&A, 203, 297

231. Verbunt, F., van Paradijs, J., \& Elson, R. 1984, MNRAS, 210, 899

232. Verbunt, F., Belloni, T., Johnston, H., van der Klis, M., \& Lewin, W. 1994, A\&A, 285, 903

233. Verbunt, F., Bunk, W., Hasinger, G., \& Johnston, H. 1995, A\&A, 300, 732

234. Verbunt, F., Kuiper, L., Belloni, T., \& et al. 1996, A\&A, 311, L9

235. Verbunt, F., Bunk, W., Ritter, H., \& Pfeffermann, E. 1997, A\&A, 327, 602

236. Verbunt, F., van Kerkwijk, M., in 't Zand, J., \& Heise, J. 2000, A\&A, 359, 960

237. Webb, N., Gendre, B., \& Barret, D. 2002, A\&A, 381, 481

238. West, M., Côté, P., Marzke, R., \& Jordán, A. 2004 Nature 427, 31

239. White, N. \& Angelini, L. 2001, ApJ, 561, L101

240. White, N. \& Ghosh, P. 1998, ApJ, 504, L31

241. White, R. 2002, APS Meeting April 2002 Abstracts 11.010

242. White, R., Sarazin, C. \& Kulkarni, S. 2002, ApJ 571, L23

243. Wijnands, R., Heinke, C., \& Grindlay, J. 2002, ApJ, 572, 1002

244. Wijnands, R., Heinke, C., \& Pooley, D., et al. 2005, ApJ, 618, 833

245. Zavlin, V., Pavlov, G., \& Shibanov, Y. 1996, A\&A, 315, 141 
246. Zepf, S. \& Ashman, K. 1993, MNRAS, 264, 611 